\title{
Relative rates of hydrogen shift isomerizations depend strongly on multiple-structure anharmonicity
}

Lili Xing, Junwei Lucas Bao, Zhandong Wang, xuetao wang, and Donald G. Truhlar

J. Am. Chem. Soc., Just Accepted Manuscript • DOI: 10.1021/jacs.8b09381 • Publication Date (Web): 23 Nov 2018

Downloaded from http://pubs.acs.org on December 2, 2018

\section{Just Accepted}

"Just Accepted" manuscripts have been peer-reviewed and accepted for publication. They are posted online prior to technical editing, formatting for publication and author proofing. The American Chemical Society provides "Just Accepted" as a service to the research community to expedite the dissemination of scientific material as soon as possible after acceptance. "Just Accepted" manuscripts appear in full in PDF format accompanied by an HTML abstract. "Just Accepted" manuscripts have been fully peer reviewed, but should not be considered the official version of record. They are citable by the Digital Object Identifier (DOI®). "Just Accepted" is an optional service offered to authors. Therefore, the "Just Accepted" Web site may not include all articles that will be published in the journal. After a manuscript is technically edited and formatted, it will be removed from the "Just Accepted" Web site and published as an ASAP article. Note that technical editing may introduce minor changes to the manuscript text and/or graphics which could affect content, and all legal disclaimers and ethical guidelines that apply to the journal pertain. ACS cannot be held responsible for errors or consequences arising from the use of information contained in these "Just Accepted" manuscripts. 


\title{
Relative rates of hydrogen shift isomerizations depend strongly on multiple-structure anharmonicity
}

Lili Xing, ${ }^{a, \dagger, *}$, Junwei Lucas Bao, ${ }^{b, \dagger}$, Zhandong Wang, ${ }^{c}$ Xuetao Wang, ${ }^{a}$ Donald G. Truhlar ${ }^{b, *}$

${ }^{a}$ Energy and Power Engineering Institute, Henan University of Science and Technology,

Luoyang, Henan 471003, China

${ }^{b}$ Department of Chemistry, Chemical Theory Center, and Minnesota Supercomputing Institute, University of Minnesota, Minnesota 55455-0431, USA

${ }^{c}$ Clean Combustion Research Center, King Abdullah University of Science and Technology, Thuwal, 23955-6900, Saudi Arabia

\begin{abstract}
Hydroperoxyalkylperoxy species (OOQOOH) are important intermediates that are generated during the autoignition of transport fuels. A key reaction of hydroperoxyalkylperoxy radicals is a [1,5] hydrogen shift, for which kinetics data are experimentally unavailable. Here we study two typical OOQOOH reactions and compare their kinetics to one another and to a previous study to learn the effect of structural variations of the alkyl group on the competition between alternative [1,5] hydrogen shifts of hydroperoxyalkylperoxy species. We use electronic structure calculations to determine previously missing thermochemical data, and we use variational transition state theory (VTST) with multidimensional tunneling (MT), multiple structures, torsional potential anharmonicity, and high-frequency anharmonicity to obtain more accurate rate constants than the ones that can computed by conventional single-structure harmonic transition state theory (TST) and than the empirically estimated rate constants that are currently used in combustion modeling. The calculated temperature range is $298-1500 \mathrm{~K}$. The roles of various factors in determining the rates are elucidated, and we find an especially strong effect of multiple structure anharmonicity due to torsions. Thus, even though there is some cancellation between the anharmonicity of the reactant and the anharmonicity of the transition state, and even though the reactants are very similar in structure, differing only by a methyl group, the effect of multiple structure anharmonicity has a large effect on the relative rates - as large as a factor of 17 at room temperature and as large as a factor of 7 at $1500 \mathrm{~K}$. This has broad implications for the estimation of reaction rates in many subfields of chemistry, including combustion chemistry and atmospheric chemistry, where rates of reaction of complex molecules are usually estimated without explicit consideration of this fundamental entropic effect. In addition, the pressure-dependence of the rate constants is modeled by system-specific quantum Rice-Ramsperger-Kassel (SS-QRRK) theory for a reversible isomerization.
\end{abstract}

KEYWORDS: Anharmonicity; Autoignition; Combustion; Entropy; Hydroperoxyalkylperoxy; Kinetics; Quantum chemical calculations; Torsions 


\section{INTRODUCTION}

Most textbook discussions of chemical kinetics involve the vibrational-rotational partition functions of reactants and the transition state, ${ }^{1}$ but recent work has shown that one may obtain quite different results if one includes all the conformations of the reactants and the transition states and calculates conformational-vibrational-rotation partition functions. ${ }^{2-6}$ This may be considered an anharmonic effect since a harmonic system has only one local minimum in the potential energy surface; it is also an entropic effect. In the present work we examine the dependence of this multiple-structure effect on the structure of the reactants by comparing the calculated rate constants for two [1,5] hydrogen shift reactions, one involving a 3-peroxy-1-hexyl group and the other differing only in that this is replaced by a 3-peroxy-5-methyl-1-hexyl group. The reactions of these species are broadly representative of a class of [1,5] hydrogen shift reactions, which are important in many subfields of chemistry, and in addition they are of specific interest as key reaction types in the so called second oxidation process of autoignition.

The autoignition chemistry of hydrocarbon fuels plays an important role in combustion chemistry. ${ }^{7,8}$ Autoignition is very sensitive to the reaction kinetics of individual steps in the mechanism, and it is strongly affected by the competition between radical chain propagation, branching, and chain termination ${ }^{9-12}$. The low-temperature autoignition of hydrocarbon fuels ${ }^{13-22}$ is initiated by abstraction of an $\mathrm{H}$ atom [reaction (i)], preferentially from the weakest $\mathrm{C}-\mathrm{H}$ bond.

$$
\mathrm{RH}+\mathrm{Y} \rightarrow \mathrm{R}+\mathrm{YH}
$$

Here R denotes $\mathrm{C}_{n} \mathrm{H}_{2 n+1}$, and $\mathrm{Y}$ denotes $\mathrm{O}_{2}, \mathrm{OH}$, or another radical. Barrierless addition of $\mathrm{O}_{2}$ to the radical $\mathrm{R}$ produces an alkylperoxy radical ROO (ii), which in turn drives the sequence of the following reactions:

$$
\begin{gathered}
\mathrm{R}+\mathrm{O}_{2} \leftrightarrow \mathrm{ROO} \\
\mathrm{ROO} \rightarrow \text { alkene }+\mathrm{HO}_{2} \\
\mathrm{ROO} \leftrightarrow \text { QOOH } \\
\mathrm{QOOH} \rightarrow \text { aldehyde }+\mathrm{OH} \\
\mathrm{QOOH} \rightarrow \text { cyclic ether }+\mathrm{OH} \\
\mathrm{QOOH} \rightarrow \text { alkene }+\mathrm{HO}_{2} \\
\mathrm{QOOH}+\mathrm{O}_{2} \leftrightarrow \text { OOQOOH } \\
\text { OOQOOH } \leftrightarrow \text { HOOQ'OOH } \\
\text { HOOQ'OOH } \rightarrow \mathrm{OH}+\text { ketohydroperoxide } \\
\text { ketohydroperoxide } \rightarrow \text { OH }+ \text { alkoxy radical }
\end{gathered}
$$

where QOOH is a carbon-centered hydroperoxyalkyl radical $\left(\mathrm{Q}=\mathrm{C}_{n} \mathrm{H}_{2 n}\right),{ }^{23} \mathrm{OOQOOH}$ is an oxygen-centered hydroperoxyalkyl peroxy radical ${ }^{10-12}$, and $\mathrm{Q}^{\prime}$ denotes $\mathrm{C}_{n} \mathrm{H}_{2 n-1}$. The most favorable isomerization path of the oxygen-centered OOQOOH radical is usually assumed to be the transfer of an $\mathrm{H}$ from the carbon attached to the hydroperoxyl group ${ }^{24,25}$, which is a particular case of step (ix); it is conventional to label this site as $\alpha$ and to call this an $\alpha-\mathrm{H}$ shift. This yields $\mathrm{HOOQ}^{\prime} \mathrm{OOH}$, and this carbon-centered radical can decompose to yield more $\mathrm{OH}$ radicals, which are known to lead to chain branching.

The fate of hydroperoxyalkylperoxy radicals QOOH and OOQOOH is of central importance to the autoignition chemistry in combustion (e.g. 500-800 K), ${ }^{9,26}$ and it is largely responsible for 
the variation of the mechanism with the size and structure of the original fuel molecule. The importance for understanding atmospheric and combustion chemistry of the role of mechanistic branching in internal hydrogen abstraction reactions, the high sensitivity to the theoretical treatment of internal rotors and pressure effects on mechanistic branching, and problems of lowtemperature oxidation mechanisms with inadequate QOOH, OOQOOH chemistry has recently been emphasized. ${ }^{26}$ However, some kinetic models that have been used have assumed that the rate constants for OOQOOH isomerization can be obtained from those for ROO isomerization by applying a 3-4 kcal/mol correction to the activation energy to account for the weaker $\mathrm{C}-\mathrm{H}$ bond on $\mathrm{Q}$ as compared to that on $\mathrm{R} .{ }^{27}$ It is convenient but risky to use this rule for OOQOOH chemistry due to the existence of multiple structures and torsional potential anharmonicity, especially for long-chain alkane systems. Furthermore, there are no experimental data for the kinetics of $\mathrm{OOQOOH}$ species because they are very reactive, and so it is impossible to test this assumption by comparison to experiment. However, this gap in our knowledge of OOQOOH chemistry can be filled by accurate thermodynamics and kinetics calculations.

An issue that complicates the situation is that some recent studies have indicated that it is also possible for a hydrogen atom to migrate from another site (different from the $\alpha$ site) of OOQOOH. ${ }^{24,28,29}$ The competition between $\alpha-H$ isomerization reactions and alternative isomerization has a large effect on the ignition delay ${ }^{27,30}$ and the possible production of highly oxygenated intermediates. ${ }^{31}$ The competition clearly depends on the precise identity of the alkane being oxidized, and the experimental study by Wang et al. indicates that highly oxygenated intermediates with one or more hydroperoxy groups are prominent in the autooxidation of various oxygenated organic compounds. ${ }^{31}$

As engine technologies further advance and new fuels emerge, there continues to be a need for more accurate predictions over a wider range of conditions and for more diverse fuel structures and formulations, and so there is a need to sort out the above issues. The competition between possible hydrogen shift isomerizations depends strongly on the difference in bond strengths of $\mathrm{C}-\mathrm{H}$ bonds at primary, secondary, and tertiary carbons, on the distance of the carbon-hydrogen bond from a radical site, and on the size of the ring in the transition state (TS) for the hydrogen shift.

In a previous paper ${ }^{32}$ we presented accurate calculations of the thermodynamics and kinetics of hydroperoxyalkylperoxy radical (a OOQOOH species), in particular 3-peroxy-1pentyl hydroperoxide, which will be labeled as reactant $\mathbf{C}$ in the present article. In the present work, we consider two other representative OOQOOH species, labeled $\mathbf{A}$ and $\mathbf{B}$, in particular, respectively, 3-peroxy-1-hexyl hydroperoxide and 3-peroxy-5-methyl-1-hexyl hydroperoxide. As shown in Scheme 1, they differ from $\mathbf{C}$ by the extension and branching of the alkyl group. 


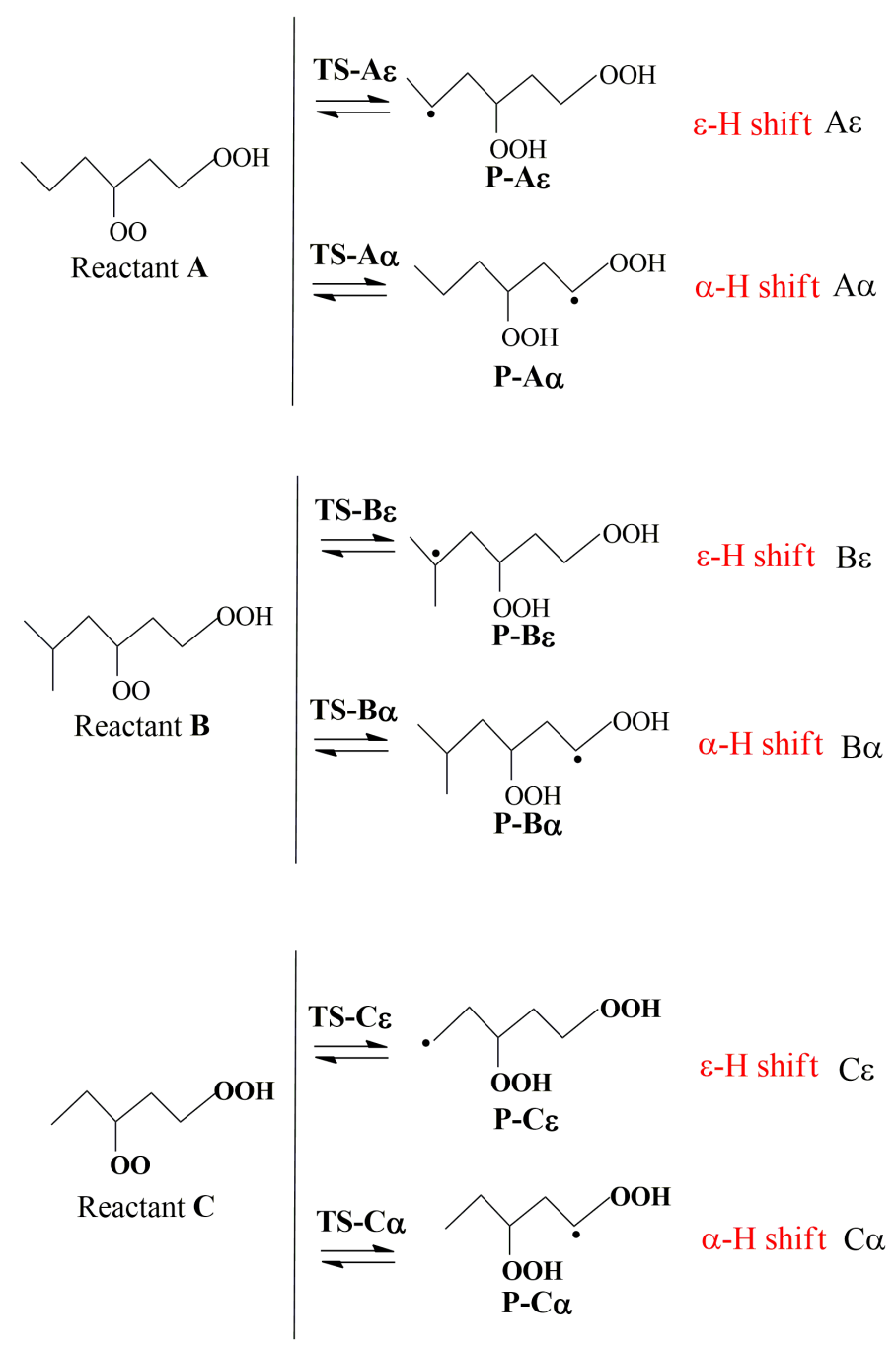

Scheme 1. Three prototypical OOQOOH species and the corresponding reactions of $[1,5] \mathrm{H}$-shift. A and $\mathbf{B}$ are studied here and $\mathbf{C}$ are studied in the previous article ${ }^{32}$.

Scheme 1 shows the $\alpha-\mathrm{H}$ shift isomerization and the competitive isomerization reactions that we consider. The $\alpha-H$ shifts are both [1,5] hydrogen shifts - thus both reactions have sixmembered rings in the $\mathrm{H}$-shift transition state. Furthermore, the $\alpha-\mathrm{H}$ position is at a secondary carbon in each of the three selected cases ( $\mathbf{A}$ and $\mathbf{B}$ studied here and $\mathbf{C}$ in the previous article ${ }^{32}$ ). In the present work we do not consider possible 1,4 and 1,6 H-shifts that have five- and sevenmembered-ring transition states. The competitive reaction paths that we consider are the other possible $[1,5]$ hydrogen shifts that have six-membered rings in the H-shift transition state. In both cases these are $\varepsilon$-H shifts; however, the competitive isomerizations differ in that the hydrogen atom transferred is at secondary carbon for reactant $\mathbf{A}$, at a tertiary carbon for reactant $\mathbf{B}$, and at primary carbon for species $\mathbf{C}$. The key question examined is: how will the competition change due to these changes in the structure of the alkyl group? 
In the rest of this article, we label products of the $\boldsymbol{\varepsilon}-\mathrm{H}$ shifts as $\mathbf{P}-\mathbf{A} \boldsymbol{\varepsilon}$ and $\mathbf{P}-\mathbf{B} \boldsymbol{\varepsilon}$ and the products of the $\boldsymbol{\alpha}-\mathrm{H}$ shifts as $\mathbf{P}-\mathbf{A} \boldsymbol{\alpha}$ and $\mathbf{P}-\mathbf{B} \boldsymbol{\alpha}$, as indicated in Scheme 1. The reactions themselves will simply be labeled $\mathrm{A} \varepsilon, \mathrm{B} \varepsilon, \mathrm{A} \alpha$, and $\mathrm{B} \alpha$.

We note that these are just a subset of the possible isomerizations. We have also calculated the forward barrier heights for the competing isomerizations of $\mathbf{A}$ and $\mathbf{B}$, and these are shown in Scheme 2. We see that $A \varepsilon$ has the lowest barrier for $\mathbf{A}$, and $\mathrm{B} \varepsilon$ has the lowest barrier for $\mathbf{B}$, even when all five possible isomerizations are considered in each case. Furthermore, two of the three other isomerizations of $\mathbf{A}$ have higher barrier heights than both of isomerizations studied here, and all three of the other isomerizations of $\mathbf{B}$ have higher barrier heights than the ones studied here.
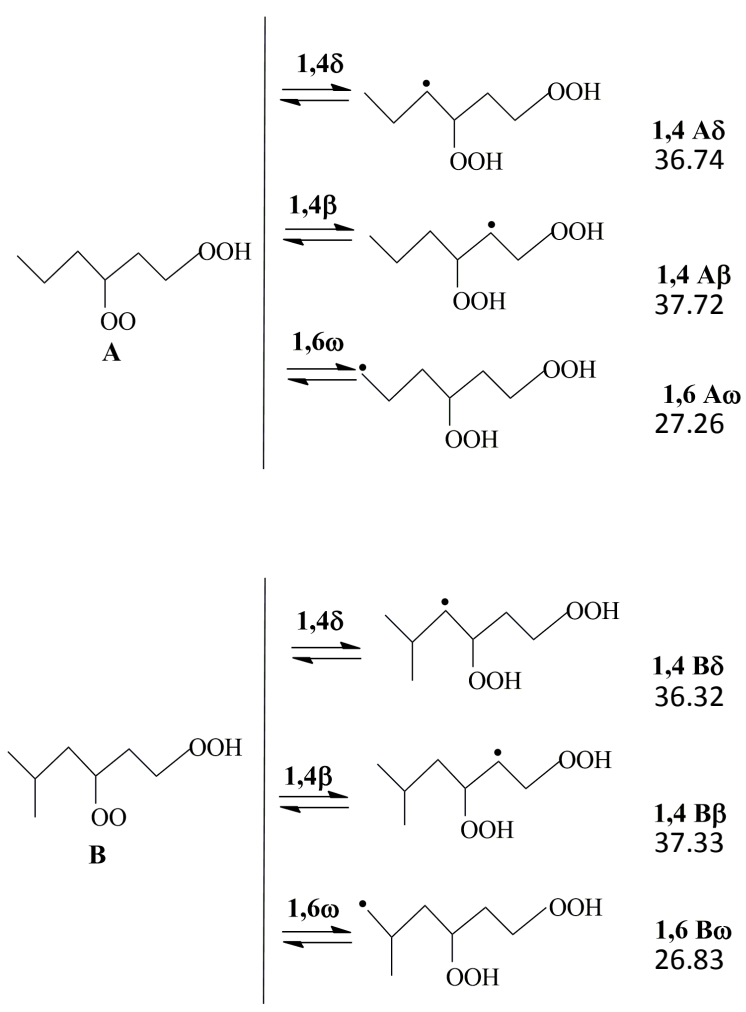

Scheme 2. Other possible isomerization channels and their forward barrier heights in $\mathrm{kcal} / \mathrm{mol}$.

The chain radicals $\mathbf{A}$ and $\mathbf{B}$ have many conformers due to internal rotations about the $\mathrm{C}-\mathrm{C}$, $\mathrm{C}-\mathrm{O}$, and $\mathrm{O}-\mathrm{H}$ bonds. ${ }^{24,33}$ Because these internal rotations are coupled, one cannot treat them adequately as independent torsions; thus we use a method capable of treating multiple conformers and coupled torsions in particular, as in the previous study ${ }^{32}$ of reactant $\mathbf{C}$, we use the multistructural method with torsional anharmonicity based on a coupled torsional potential, ${ }^{34}$ which will be abbreviated as the method. For the kinetics, we use multistructural variational transition state theory (MS-VTST) with multidimensional tunneling contributions and potential energy surfaces obtained by direct dynamics, where "direct dynamics" refer to ${ }^{35,36}$ calculating energies, gradients, and Hessians of the potential energy surfaces as needed by electronic 
structure calculations. The MS-VTST rate calculations include multiple-structure anharmonicity and torsional potential anharmonicity. To compute the pressure dependence of the thermal rate constants, we use system-specific quantum Rice-Ramsperger-Kassel (SS-QRRK) theory ${ }^{37}$ with the thermal activation mechanism. ${ }^{38-40}$

\section{METHODOLOGY AND COMPUTATIONAL DETAILS}

\subsection{Overview}

First we performed an exhaustive search for the equilibrium structures of the conformers of the reactants and products and the transition structures of the conformers of the transition states. In order to make a consistent comparison with the previous study ${ }^{32}$ of the reactions of $\mathbf{C}$, we used the M05-2X exchange-correlation functional ${ }^{35}$ and the jun-cc-pVTZ ${ }^{36}$ basis set for the conformer optimization. The M05-2X/jun-cc-pVTZ method was selected in the previous work due to the high performance-cost ratio; in particular it possess a mean unsigned deviation of $0.27 \mathrm{kcal} / \mathrm{mol}$ from the benchmark results in the previous study, ${ }^{32}$ and its good performance is reconfirmed below for the present reactions. Frequency analysis was performed with the stationary points either as equilibrium structures with no imaginary frequencies or as transition structures with one imaginary frequency. We used the MS-T method ${ }^{40-42}$ for treating multistructural torsional anharmonicity effects.

We combined variational effects, internal-coordinate-based vibrationally adiabatic potential curves, multistructural torsional anharmonicity, and multidimensional tunneling by the MSVTST method for calculating rate constants in the high-pressure limit.

Pressure-dependent rate constants were calculated using system-specific quantum RiceRamsperger-Kassel (SS-QRRK) theory ${ }^{43}$ combined with the thermal activation mechanism, ${ }^{38-40}$ which involves competition between reaction of activated species and their collisional deactivation. SS-QRRK theory is a system-specific version ${ }^{43}$ of the original QRRK theory ${ }^{44}$ and its extensions by Dean, Westmoreland, Bozzelli et al.; ${ }^{45-48}$ SS-QRRK allows one to include multistructural anharmonicity and tunneling in a particularly efficient way, by using highpressure canonical ensemble rate constants to obtain system-specific, temperature-dependent effective threshold energies and pre-exponential Arrhenius factors for the calculation of pressure effects. All our SS-QRRK calculations are for carried out under the independent-reaction approximation in which the effect of competitive reactions, if present, on the energy distribution of activated molecules of the reactant is neglected. Within this independent-reaction approximation our previous presentation of SS-QRRK was appropriate for dissociation reactions, very exothermic isomerizations, or any isomerization where the reverse reaction is neglected. In the present case, we consider endothermic isomerizations, and we extend the formalism to include the reverse reaction in the mechanism.

\subsection{Details: high-pressure limit}

Both the SCF calculations and the geometry optimizations were performed with tight convergence criteria. The numerical integrations were performed using a grid with 99 radial shells around each atom and 974 angular points in each shell. All the electronic structure calculations were performed using the Gaussian 16 software package. ${ }^{49}$ The initial conformers 
for the structure search were generated by rotating all the torsional bonds (except for the methyl group, which does not generate distinguishable conformers) by using the MSTor program. ${ }^{41,42,50}$

Classical barrier heights for forward and reverse reactions and the classical energy of reaction were computed based on the lowest-energy structures. "Classical” energies are differences in potential energies, i.e., they include neither zero point energy nor thermal vibrational-rotational contributions. Although equilibrium constants and rate constants depend on free energies, which do include those contributions, classical energetics are used for comparing electronic structure theories.

The multistructural (MS) canonical variational theory (CVT) rate constant ${ }^{2}$ with the smallcurvature approximation for tunneling ${ }^{51}$ (SCT) is obtained by multiplying the single-structural $(\mathrm{SS})^{52} \mathrm{CVT} / \mathrm{SCT}$ rate constant by the multistructural torsional anharmonicity factor $F_{\mathrm{act}}^{\mathrm{MS}-\mathrm{T}}$, that is $^{2}$

$$
k^{\mathrm{MS}-\mathrm{CVT} / \mathrm{SCT}}=F_{\mathrm{act}}^{\mathrm{MS}-\mathrm{T}} k_{1}^{\mathrm{SS}-\mathrm{CVT} / \mathrm{SCT}}
$$

Note that $k_{1}^{\mathrm{SS}-\mathrm{CVT} / \mathrm{SCT}}$ employs the lowest-energy conformer (labeled as 1 here) for the reactant and the transition state. If calculations are done with the tunneling transmission coefficient set equal to unity (i.e., neglecting tunneling), the result is called quasiclassical. The quasiclassical CVT method variationally optimizes the location of the dividing surface to maximize the free energy of activation, and in MS-CVT, this is determined entirely by the reaction path through the lowest-energy path through the transition state, and multistructural torsional anharmonicity is estimated based on the transition structure of that path.

The multidimensional tunneling (MT) contributions for the lowest-energy path were evaluated with the small-curvature tunneling approximation $(\mathrm{SCT})^{51,53}$. The vibrationally adiabatic ground-state energy, which is the effective potential for tunneling, is given by:

$$
V_{\mathrm{a}}^{\mathrm{G}}=V_{\mathrm{MEP}}(s)+\varepsilon^{\mathrm{G}}(s)
$$

where $\varepsilon^{\mathrm{G}}(s)$ is the local zero point energy, and $s$ is the reaction coordinate, which is the progress variable along the lowest-energy path.

We used the MS-T method ${ }^{34,41,42,50,54}$, including all the inter-convertible torsional conformers, to calculate the partition functions as functions of temperature. For the reactions considered here, all of the species (reactants, transition structures, products) are chiral molecules, and thus the rate constant for a reaction can be computed based solely on the (S) structure or the (R) structure. (We have discussed this in detail in a previous article. ${ }^{54}$ ) Therefore, when counting the distinguishable conformers, the number of conformers reported is for only one of the enantiomers. The conformational-vibrational-rotation partition function at temperature $T$ of the given species (reactant, product, or transition state) with $J$ distinguishable conformers and $t$ torsions is

$$
\begin{gathered}
Q_{\text {con-rovib }}^{\mathrm{MS}-\mathrm{T}}=\sum_{j=1}^{J} Q_{j}^{\mathrm{SS}-\mathrm{T}} \\
Q_{j}^{\mathrm{SS}-\mathrm{T}}=Q_{\mathrm{rot}, j} \exp \left(-\frac{U_{j}}{k_{B} T}\right) Q_{j}^{\mathrm{QH}} \prod_{\tau=1}^{t} \widetilde{f_{j, \tau}}
\end{gathered}
$$

where $j$ denotes a conformer, $Q_{\mathrm{rot}, j}$ is the classical rotational partation function of conformer $j$ including the symmetry number for overall rotation (these symmetry numbers are unity for all cases in this article), $U_{j}$ is the relative potential energy when the local minimum of the potential 
energy surface for the calculation of the partition function of a given species is chosen as the local zero of energy, $k_{\mathrm{B}}$ is the Boltzmann constant, $Q_{j}^{\mathrm{QH}}$ is the quasiharmonic $(\mathrm{QH})$ vibrational partition function, and $\widetilde{f_{j, \tau}}$ is a factor that accounts for the potential function anharmonicity of each coupled torsion $\tau$. In eq. 4, "quasiharmonic" means the use of the harmonic oscillator formulas combined with scaled harmonic frequencies where the scaling accounts (in an approximate way) for anharmonicity of high-frequency modes and for systematic errors in the electronic structure calculations of harmonic frequencies. The scale factor or M05-2X/jun-ccpVTZ is 0.964 , as determined previously. ${ }^{55}$

Torsions are defined here by internal coordinates rather than by normal-mode coordinates. The reference potential for the torsional coordinate $\phi_{j, \tau}$ of torsion $\tau$ of structure $j$ of a given species is approximated locally as

$$
V_{j, \tau}=U_{j}+A_{j, \tau}\left[1-\cos M_{j, \tau}\left(\phi_{j, \tau}-\phi_{j, \tau, \mathrm{eq}}\right)\right]
$$

where $\phi_{j, \tau \text {,eq }}$ is the equilibrium value of the torsion angle, $M_{j, \tau}$ is the local periodicity, determined by Voronoi tessellation, and $A_{j, \tau}$ is determined from the second-order force constants and the local periodicities ${ }^{34}$. The effects caused by the deviation of eq. 5 from a harmonic potential are called torsional potential anharmonicity effects; the effect of including the contributions of all the distinguishable conformers of a species is called multiple-structure anharmonicity. The combination of these two effects is called multistructural torsional anharmonicity, and it can be characterized for species $\mathrm{X}$ by the following factor:

$$
F_{\mathrm{X}}^{\mathrm{MS}-\mathrm{T}}=\frac{Q_{\mathrm{con}-\mathrm{rovib}}^{\mathrm{MS}-\mathrm{X}}(\mathrm{X})}{Q_{\mathrm{rovib}, \mathrm{GM}}^{\mathrm{SS}-\mathrm{QH}}(\mathrm{X})}
$$

Here the denominator is the single-structural quasiharmonic rovibrational partition function for the global minimum structure of X. $F_{\mathrm{act}}^{\mathrm{MS}-\mathrm{T}}$ is defined to be the multistructural torsional anharmonicity factor of the reaction as follows:

$$
F_{\text {act }}^{\mathrm{MS}-\mathrm{T}}=F_{\mathrm{TS}}^{\mathrm{MS}-\mathrm{T}} / F_{\mathrm{R}}^{\mathrm{MS}-\mathrm{T}}
$$

It includes the contributions from all the conformational structures of the reactants and the transition state structures and is computed by the coupled-potential MS-T method as described previously $^{37-39,56}$.

For comparison we will also consider two more approximate ways to evaluate the partition functions. The MS-T result reduces to the multistructural local quasiharmonic approximation (MS-LQH) if we set all the $\widetilde{f_{j, \tau}}$ equal to unity in eq 4. If, in addition, we keep only the first term (lowest-energy structure) in eq 3, we get the single-structure-quasiharmonic (SS-QH) approximation.

All the direct dynamics calculations were performed with the Polyrate $2016-2 \mathrm{~A}^{57}$ and Gaussrate 2017 codes. $^{58}$ The minimum energy paths (MEPs) needed for the calculations were calculated in isoinertial coordinates using the Page-McIver method, which is also called the local quadratic approximation (LQA), ${ }^{59}$ and using the reorientation of the dividing surface (RODS) algorithm ${ }^{60}$. The step size was $0.0026 \AA$ (with masses scaled to $1 \mathrm{amu}$ ), and the Hessians were updated every 10 steps along the reaction path. The generalized normal mode analyses were 
performed using nonredundant internal coordinates ${ }^{61,62}$ with a scaling factor of $0.964 ;{ }^{55}$ the coordinates used for this calculation are specified in Supporting Information (SI).

\subsection{Details: pressure dependence}

Full presentations of the pressure-dependent SS-QRRK model for an irreversible unimolecular reaction are given in our previous paper $^{32}$ and in a recent review ${ }^{40}$ for the case where the mechanism assumed is to be

$$
\begin{gathered}
\mathrm{X}(T)+\mathrm{M} \underset{k_{\text {deact }}(T)}{\stackrel{k_{\text {act }}(E, T)}{\rightleftarrows}} \mathrm{X}^{*}(E)+\mathrm{M} \\
\mathrm{X}^{*}(E) \stackrel{k_{\mathrm{con}, \mathrm{X}}(E)}{\longrightarrow} \mathrm{P}
\end{gathered}
$$

where $\mathrm{X}$ is the reactant, $\mathrm{M}$ is bath gas, $\mathrm{P}$ is the product, and $\mathrm{X}^{*}$ is the rovibrationally excited (energized) complex, which is generated by collisions with a thermal ensemble of bath molecules M. This leads to the central QRRK equation (eq 158 in the review ${ }^{40}$ ), which is

$$
k_{\text {uni }}(T, p)=\sum_{n=m}^{+\infty} \frac{(p / R T) k_{\text {act }}(n, T) k_{\text {con }, \mathrm{X}}(n ; m ; A)}{(p / R T) k_{\text {deact }}(T)+k_{\text {con }, \mathrm{X}}(n ; m ; A)}
$$

where

$$
\begin{gathered}
n=E / h c \bar{v} \\
m=E_{0} / h c \bar{v}
\end{gathered}
$$

where $p$ is pressure, $R$ is the gas constant, $E$ is internal energy, $E_{0}$ is the threshold energy, $A$ is the Kassel frequency factor, $h$ is Planck's constant, $c$ is the speed of light, and $\bar{v}$ is the geometric mean vibrational frequency in wave numbers.

In SS-QRRK theory, $k_{\text {con,X }}(n ; m ; A)$ is evaluated by eq 162 of the review article, ${ }^{40}$ which is

$$
k_{\mathrm{con}, \mathrm{X}}(n ; m(T) ; A(T))=A(T) \frac{n !(n-m(T)+s-1) !}{(n-m(T)) !(n+s-1) !}
$$

where

$$
m(T)=E_{0}(T) / h c \bar{v}
$$

and where $A(T)$ and $E_{0}(T)$ are evaluated by Arrhenius analysis of the thermal rate constant. Thus in SS-QRRK theory, eq 8 is replaced by

$$
k_{\text {uni }}(T, p)=\sum_{n=m(T)}^{+\infty} \frac{(p / R T) k_{\text {act }}(n, T) k_{\mathrm{con}, \mathrm{X}}(n ; m(T) ; A(T))}{(p / R T) k_{\text {deact }}(T)+k_{\mathrm{con}, \mathrm{X}}(n ; m(T) ; A(T))}
$$

The assumption that dissociation of the activated molecule in step S2 is irreversible is a good approximation for dissociative reactions (i.e., when $\mathrm{P}$ corresponds to two or more fragments) and for strongly exothermic isomerizations. ${ }^{63}$ However, it is not necessarily valid for endothermic or slightly exothermic reactions where the nascent energized product $\mathrm{P}^{*}$ has a high probability of reverting back to energized reactant $\mathrm{R}^{*}$. In such cases one must consider, instead of step S2, the reversible reaction 


$$
\mathrm{X}^{*}(E) \underset{k_{\text {con }, \mathrm{P}}(E)}{\stackrel{k_{\mathrm{con}, \mathrm{X}}(E)}{\rightleftarrows}} \mathrm{P} * \stackrel{k_{\text {deact }}^{\prime}(T)}{\longrightarrow} \mathrm{P}
$$

Equation 13 is analogous to the central equation of RRKM theory, and Lin and Laidler ${ }^{64}$ (see also Kiefer et al. ${ }^{65}$ ) have shown that accounting for this reversibility by replacing step S2 with step S2rev changes the steady-state rate in RRKM theory. Applying their steady-state treatment to SS-QRRK theory replaces eq 13 by

$$
k_{\text {uni }}(T, p)=\sum_{n=m(T)}^{+\infty} \frac{(p / R T) k_{\mathrm{act}}(n, T) k_{\mathrm{con}, \mathrm{X}}(n ; m(T) ; A(T))}{(p / R T) k_{\text {deact }}(T)+k_{\mathrm{con}, \mathrm{X}}(n ; m(T) ; A(T))+k_{\mathrm{con}, \mathrm{P}}\left(n ; m^{\prime}(T) ; A^{\prime}(T)\right) \frac{k_{\text {deact }}(T)}{k_{\text {deact }}^{\prime}(T)}}
$$

where

$$
\begin{aligned}
n^{\prime} & =(E-\Delta E) / h c \bar{v} \\
m^{\prime} & =\left(E_{0}-\Delta E\right) / h c \bar{v}
\end{aligned}
$$

and $\Delta E$ is the energy of reaction (positive for an endoergic reaction). If we make the reasonable assumptions that $k_{\text {deact }}(T)$ equals $k_{\text {deact }}^{\prime}(T)$, this reduces to

$$
k_{\text {uni }}(T, p)=\sum_{n=m(T)}^{+\infty} \frac{(p / R T) k_{\mathrm{act}}(n, T) k_{\mathrm{con}, \mathrm{X}}(n ; m(T) ; A(T))}{(p / R T) k_{\mathrm{deact}}(T)+k_{\mathrm{con}, \mathrm{X}}(n ; m(T) ; A(T))+k_{\mathrm{con}, \mathrm{P}}\left(n^{\prime} ; m^{\prime}(T) ; A^{\prime}(T)\right)}
$$

In the absence of a thermal rate constant calculation for the reverse reaction, we may assume $A^{\prime}(T)$ is the same as $A(T)$. Since $\Delta E$ is known, we can then evaluate $k_{\text {con,P }}\left(n^{\prime} ; m^{\prime}(T) ; A(T)\right)$ by eqs 11,15 , and 16 .

It is instructive to rewrite eq 17 as

$$
k_{\text {uni }}(T, p)=\sum_{n=m(T)}^{+\infty} \frac{\left(k_{\text {act }}(n, T) / k_{\text {deact }}(T)\right) k_{\mathrm{con}, \mathrm{X}}(n ; m(T) ; A(T))}{1+\frac{k_{\mathrm{con}, \mathrm{X}}(n ; m(T) ; A(T))+k_{\mathrm{con}, \mathrm{P}}\left(n^{\prime} ; m^{\prime}(T) ; A^{\prime}(T)\right)}{(p / R T) k_{\text {deact }}(T)}}
$$

which differs from the result for a reversible reaction by the second term in the numerator of the fraction in the denominator. This term is negligible as compared to the first term for a strongly exothermic reaction, but not otherwise. We also note that the fraction in the numerator is the equilibrium constant for reaction M1, and the fraction in the denominator vanishes in the highpressure limit. When one is not in the high-pressure limit, the reversibility lowers the rate constant due to reversion of nascent $\mathrm{P}^{*}$ molecules to reactants before they can be deactivated.

In applying eq 17 (or equivalently 18), we set $\Delta E$ in eqs 15 and 16 equal to the enthalpy of reaction at $0 \mathrm{~K}$ (this involves correcting the $\Delta E$ of Table 1 for the difference of zero point energy in the product and reactant).

The Lennard-Jones parameters needed to calculate the collisional de-activation rate constants were estimated according to empirical formulae. ${ }^{66}$ For species $\mathbf{A}, \sigma_{l}=6.51 \AA, \varepsilon_{l} / k_{\mathrm{b}}=$ $505.9 \mathrm{~K}$; for species $\mathbf{B}, \sigma_{2}=6.82 \AA, \varepsilon_{2} / k_{\mathrm{b}}=524.9 \mathrm{~K}$; and for the bath gas $\mathrm{Ar}, \sigma_{3}=3.47 \AA$ and $\varepsilon_{3} / k_{\mathrm{b}}=114.0 \mathrm{~K}$. The average energy transferred per deactivating collision, which determines the 
collision efficiency and plays an important role in the falloff effect, ${ }^{67}$ is evaluated by the simple power law representation: ${ }^{68-70}\langle\Delta E\rangle_{\text {down }}=\Theta(\mathrm{T} / 300)^{0.85}$. (Do not confuse the energy transfer $\Delta E$ in the collisional deactivation step with the energy of reaction in the equations above.) In the present work, the empirical value $\Theta$ is estimated as $600 \mathrm{~cm}^{-1}$ for $A \varepsilon$ and $A \alpha$ and $800 \mathrm{~cm}^{-1}$ for $B \varepsilon$ and $\mathrm{B} \alpha$, which is based on the empirical rule that "larger species generally having larger values," ${ }^{, 68-70}$ and we also made checks of the sensitivity to this parameter. The formulas relating the collisional deactivation rate constants $k_{\mathrm{c}}$ to $\langle\Delta E\rangle_{\text {down }}$ are given elsewhere. ${ }^{38,71}$

\section{RESULTS AND DISCUSSION}

\subsection{Electronic structure calculations}

We performed benchmark calculations by the explicitly correlated electronic structure method CCSD(T)-F12a $\mathrm{a}^{72,73}$ with the jun-cc-pVDZ ${ }^{36}$ basis set; the combination of F12a with this basis set should be within about $0.5 \mathrm{kcal} / \mathrm{mol}$ of the complete-basis-set limit. ${ }^{36}$ Table 1 gives the classical barrier heights (forward $V_{f}$ and reverse $V_{r}$ ) and energies of reaction $(\Delta E)$ for reactions $\mathrm{A} \varepsilon, \mathrm{A} \alpha, \mathrm{B} \varepsilon$ and $\mathrm{B} \alpha$ calculated by the M05-2X/jun-cc-pVTZ method. The mean unsigned deviations (MUD) from the benchmark calculations are also listed in Table 1. The MUDs of the density functional calculations agree with benchmark results within an average of 0.4-0.5 $\mathrm{kcal} / \mathrm{mol}$.

Table 1. Classical forward $\left(V_{f}\right)$ and reverse $\left(V_{r}\right)$ barriers and energy of reaction $(\Delta E)$ in $\mathrm{kcal} / \mathrm{mol}$; mean unsigned deviations (MUDs, in $\mathrm{kcal} / \mathrm{mol}$ ) from the benchmark $^{a}$

\begin{tabular}{|c|c|c|c|c|c|c|c|}
\hline & $V_{f}$ & $V_{r}$ & $\Delta E$ & $V_{f}$ & $V_{r}$ & $\Delta E$ & $\operatorname{MUD}^{b}$ \\
\hline & & $A \varepsilon$ & & & $A \alpha$ & & \\
\hline CCSD(T)-F12a/jun-cc-pVDZ & 25.22 & 11.84 & 13.39 & 27.16 & 17.78 & 9.38 & \\
\hline \multirow[t]{2}{*}{ M05-2X/jun-cc-pVTZ } & 25.34 & 11.94 & 13.40 & 27.31 & 16.89 & 10.41 & 0.41 \\
\hline & & $\mathrm{B} \varepsilon$ & & & $\mathrm{B} \alpha$ & & \\
\hline CCSD(T)-F12a/jun-cc-pVDZ & 23.00 & 11.73 & 11.28 & 24.89 & 16.00 & 8.89 & \\
\hline M05-2X/jun-cc-pVTZ & 23.20 & 12.16 & 11.04 & 25.16 & 15.37 & 9.79 & 0.48 \\
\hline
\end{tabular}

${ }^{a}$ All energies in this table are computed at M05-2X/ jun-cc-pVTZ geometries.

${ }^{b}$ Mean unsigned deviation from $\operatorname{CCSD}(\mathrm{T})-\mathrm{F} 12 \mathrm{a} / \mathrm{jun}-\mathrm{cc}-\mathrm{pVDZ}$ for reaction energies and forward and reverse barrier heights.

Reactants A and B each have seven torsions (excluding internal rotations of methyl groups), and M05-2X/jun-cc-pVTZ calculations on these species yield respectively 323 and 260 distinguishable structures with energy distributions shown in Figure 1. The numbers of

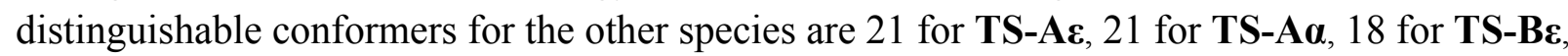
25 for TS-Ba, 300 for $\mathbf{P}-\mathbf{A \varepsilon}, 304$ for $\mathbf{P}-\mathbf{A \alpha}, 287$ for $\mathbf{P}-\mathbf{B} \boldsymbol{\varepsilon}$ and 90 for $\mathbf{P}-\mathbf{B} \boldsymbol{\alpha}$. The much lower numbers of structures for the transition states than the reactants or products is due to the ring structure characteristic of the transition states for the isomerizations. As a consequence, the conformational-vibrational-rotational transition state partition functions are smaller than those of the reactant, which will decreases the rate constants. 


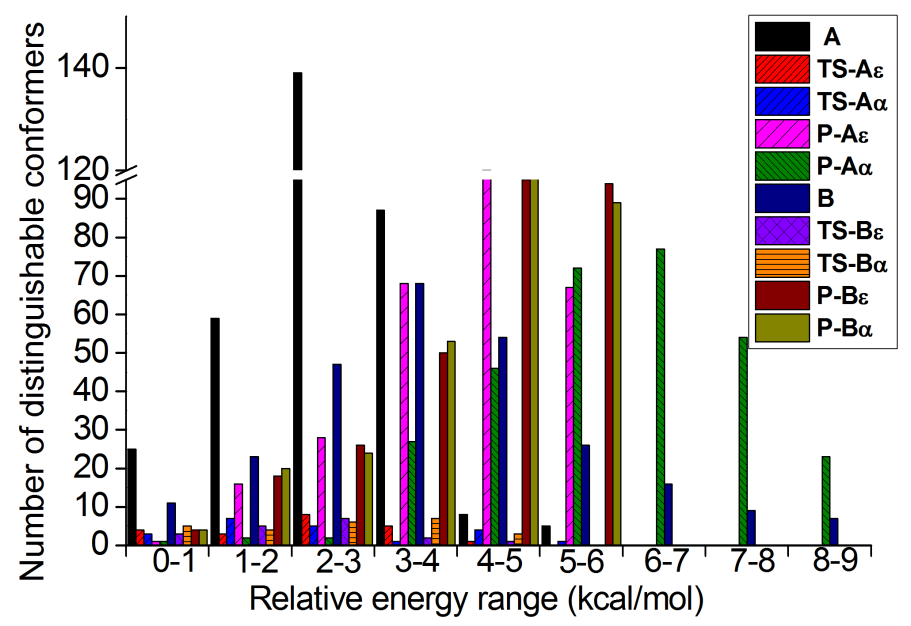

Fig. 1 Number of distinguishable conformers in each relative potential energy range for reactants

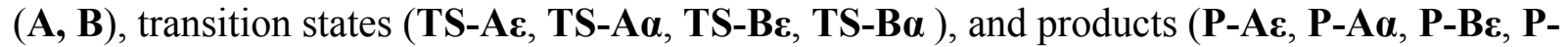
$\mathbf{B} \boldsymbol{\alpha})$. For each stable species, the energy of the global-minimal structure is chosen as the zero of the relative energy for this figure; for the transition states the energies are relative to the lowestenergy transition structure for that reaction.

\subsection{Multistructural Torsional Anharmonicity}

A major concern in modeling this kind of reaction is that internal hydrogen bonding changes the weights of the various conformational structures. We note that even though the energy of an $\mathrm{H}$-bonded structure may be lower than that of non-H-bonded structures, its free energy may be higher due primarily to the angular constraint of the $\mathrm{H}$ bond (for example, the H-bonded structure may be a ring structure and the non-H-bonded structure may be an open chain). A key feature of the methods used here is that we use a density functional that has good performance for hydrogen bonding and conformational energies, and we include all structures of the reactant and the transition state, correctly weighted according to their free energy, whether they are hydrogen bonded or not.

The local periodicities of structures of the reactants $\mathbf{A}$ and $\mathbf{B}$, products $(\mathbf{P}-\mathbf{A} \boldsymbol{\varepsilon}, \mathbf{P}-\mathbf{A} \boldsymbol{\alpha}, \mathbf{P}-\mathbf{B} \boldsymbol{\varepsilon}$,

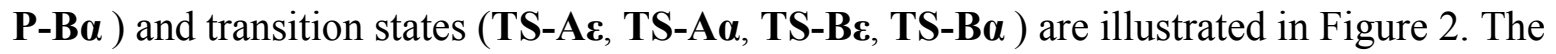
physical meaning of the $M_{j, \tau}$ parameter was explained in detail in the original MS-T paper, ${ }^{74}$ but a rough explanation of the $M_{j, \tau}$ parameters is that they tell us the effective numbers of minima along the torsional coordinates. Figure 2 shows that the local periodicities usually spread over the range of 1.7-2.8. In addition to the issue of variable steric strain, one reason why we see a variety of $M_{j, \tau}$ values in Figure 2 is the role of hydrogen bonding in some but not all of the structures. 


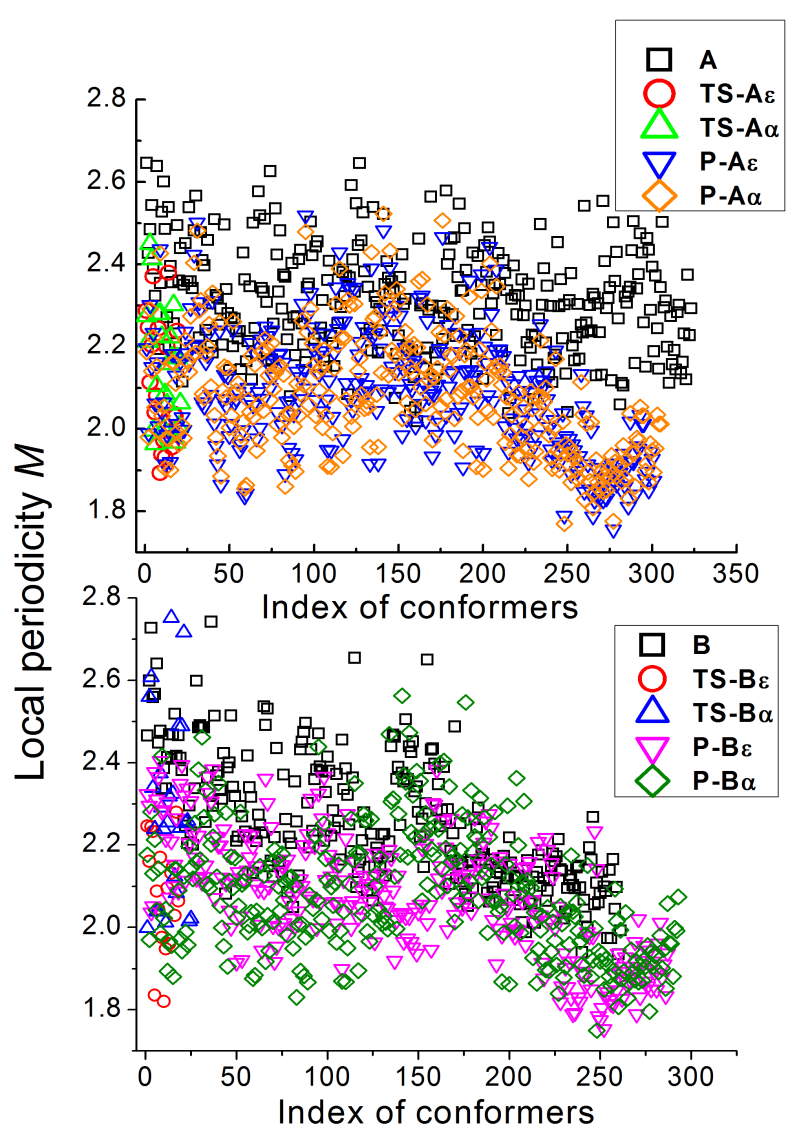

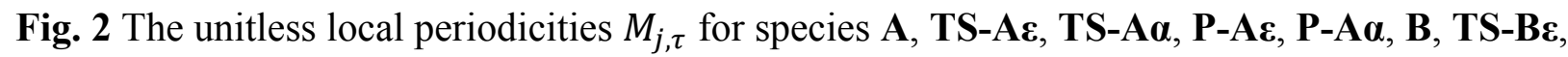
TS-B $\boldsymbol{\alpha}, \mathbf{P}-\mathbf{B} \boldsymbol{\varepsilon}$, and P-B $\boldsymbol{\alpha}$.

We employed eqs. 3 and 4 to calculate the conformational-rotational-vibrational partition functions of the reactants $\mathbf{A}$ and $\mathbf{B}$, products (P-A\&, P-A $\boldsymbol{\alpha}, \mathbf{P}-\mathbf{B} \boldsymbol{\varepsilon}, \mathbf{P}-\mathbf{B} \boldsymbol{\alpha})$ and transition states (TS-

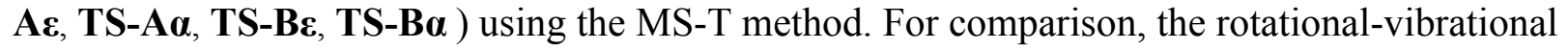
partition function of the lowest-energy structure was also calculated using the single-structure quasiharmonic oscillator approximation (SS-QH). The calculated partition functions of the reactant and transition state are given in Table 2. Note that -because we put the zero of energy for the partition functions at the lowest potential energy rather than at the zero-point level - these partition functions include the Boltzmann factor of the zero point energy, and thus they include the full effect of vibrational energy, rotational energy, and higher-energy conformations, not just the effect of thermal excitation above the ground state. The MS-T method is more accurate than either the SS-QH or MS-LQH approximations because it includes both multistructural and torsional potential anharmonicities. For reactant $\mathbf{A}$ the conformational-rotational-vibrational partition functions by the MS-T method are greater than the SS-QH ones by factors of 217-3371, and for reactant B, they are 40-1049 greater than the SS-QH ones, where the ranges reflect that these ratios increase with temperature. These results show that there is a huge difference between the MS-T and SS-QH methods. 
Table 2. Conformational-rotational-vibrational functions of the reactants $(\mathbf{A}, \mathbf{B})$ and transition

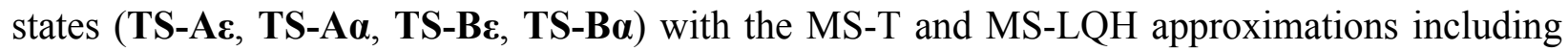
all structures and the SS-QH method for the lowest-energy structure ${ }^{\mathrm{a}}$

\begin{tabular}{|c|c|c|c|c|c|c|c|}
\hline & $T(\mathrm{~K})$ & $\bar{A}$ & TS-AE & TS-A $\alpha$ & $\bar{B}$ & TS-BE & TS-Ba \\
\hline \multirow{7}{*}{$Q_{\text {con-rovib }}^{\mathrm{MS}-\mathrm{T}}$} & 298 & $1.59 \times 10^{-76}$ & $1.36 \times 10^{-75}$ & $1.64 \times 10^{-75}$ & $6.48 \times 10^{-89}$ & $2.50 \times 10^{-87}$ & $1.58 \times 10^{-87}$ \\
\hline & 400 & $3.42 \times 10^{-52}$ & $4.56 \times 10^{-52}$ & $3.94 \times 10^{-52}$ & $3.63 \times 10^{-61}$ & $2.10 \times 10^{-60}$ & $1.09 \times 10^{-60}$ \\
\hline & 600 & $5.32 \times 10^{-27}$ & $1.10 \times 10^{-27}$ & $6.62 \times 10^{-28}$ & $2.03 \times 10^{-32}$ & $1.63 \times 10^{-32}$ & $7.12 \times 10^{-33}$ \\
\hline & 800 & $4.39 \times 10^{-13}$ & $3.46 \times 10^{-14}$ & $1.79 \times 10^{-14}$ & $1.60 \times 10^{-16}$ & $4.38 \times 10^{-17}$ & $1.84 \times 10^{-17}$ \\
\hline & 1000 & $8.22 \times 10^{-4}$ & $3.60 \times 10^{-5}$ & $1.74 \times 10^{-5}$ & $6.36 \times 10^{-6}$ & $8.71 \times 10^{-7}$ & $3.70 \times 10^{-7}$ \\
\hline & 1200 & $5.92 \times 10^{3}$ & $1.75 \times 10^{2}$ & $8.18 \times 10^{1}$ & $4.42 \times 10^{2}$ & $3.73 \times 10^{1}$ & $1.62 \times 10^{1}$ \\
\hline & 1500 & $3.27 \times 10^{11}$ & $6.51 \times 10^{9}$ & $2.98 \times 10^{9}$ & $3.19 \times 10^{11}$ & $1.62 \times 10^{10}$ & $7.26 \times 10^{9}$ \\
\hline \multirow{7}{*}{$Q_{\text {con-rovib }}^{\mathrm{MS}-\mathrm{LQH}}$} & 298 & $1.83 \times 10^{-76}$ & $1.95 \times 10^{-75}$ & $3.95 \times 10^{-75}$ & $8.29 \times 10^{-89}$ & $3.46 \times 10^{-87}$ & $2.91 \times 10^{-87}$ \\
\hline & 400 & $3.50 \times 10^{-52}$ & $5.82 \times 10^{-52}$ & $8.72 \times 10^{-52}$ & $4.04 \times 10^{-61}$ & $2.57 \times 10^{-60}$ & $1.84 \times 10^{-60}$ \\
\hline & 600 & $4.61 \times 10^{-27}$ & $1.23 \times 10^{-27}$ & $1.33 \times 10^{-27}$ & $1.81 \times 10^{-32}$ & $1.70 \times 10^{-32}$ & $1.06 \times 10^{-32}$ \\
\hline & 800 & $3.46 \times 10^{-13}$ & $3.71 \times 10^{-14}$ & $3.43 \times 10^{-14}$ & $1.24 \times 10^{-16}$ & $4.27 \times 10^{-17}$ & $2.56 \times 10^{-17}$ \\
\hline & 1000 & $6.25 \times 10^{-4}$ & $3.85 \times 10^{-5}$ & $3.29 \times 10^{-5}$ & $4.59 \times 10^{-6}$ & $8.42 \times 10^{-7}$ & $4.97 \times 10^{-7}$ \\
\hline & 1200 & $4.52 \times 10^{3}$ & $1.91 \times 10^{2}$ & $1.56 \times 10^{2}$ & $3.11 \times 10^{2}$ & $3.70 \times 10^{1}$ & $2.18 \times 10^{1}$ \\
\hline & 1500 & $2.65 \times 10^{11}$ & $7.55 \times 10^{9}$ & $5.97 \times 10^{9}$ & $2.30 \times 10^{11}$ & $1.73 \times 10^{10}$ & $1.02 \times 10^{10}$ \\
\hline \multirow{7}{*}{$Q_{\mathrm{rovib}, 1}^{\mathrm{SS}-\mathrm{QH}}$} & 298 & $7.32 \times 10^{-79}$ & $1.35 \times 10^{-76}$ & $9.07 \times 10^{-76}$ & $1.63 \times 10^{-90}$ & $7.85 \times 10^{-89}$ & $7.01 \times 10^{-88}$ \\
\hline & 400 & $7.88 \times 10^{-55}$ & $2.23 \times 10^{-53}$ & $1.59 \times 10^{-52}$ & $4.34 \times 10^{-63}$ & $3.66 \times 10^{-62}$ & $3.17 \times 10^{-61}$ \\
\hline & 600 & $5.02 \times 10^{-30}$ & $2.23 \times 10^{-29}$ & $1.71 \times 10^{-28}$ & $8.83 \times 10^{-35}$ & $1.31 \times 10^{-34}$ & $1.11 \times 10^{-33}$ \\
\hline & 800 & $2.47 \times 10^{-16}$ & $4.30 \times 10^{-16}$ & $3.43 \times 10^{-15}$ & $3.71 \times 10^{-19}$ & $2.28 \times 10^{-19}$ & $1.92 \times 10^{-18}$ \\
\hline & 1000 & $3.38 \times 10^{-7}$ & $3.34 \times 10^{-7}$ & $2.73 \times 10-^{6}$ & $9.85 \times 10^{-9}$ & $3.52 \times 10^{-9}$ & $2.95 \times 10^{-8}$ \\
\hline & 1200 & $2.02 \times 10^{0}$ & $1.36 \times 10^{0}$ & $1.12 \times 10^{1}$ & $5.28 \times 10^{-1}$ & $1.30 \times 10^{-1}$ & $1.09 \times 10^{0}$ \\
\hline & 1500 & $9.70 \times 10^{7}$ & $4.39 \times 10^{7}$ & $3.67 \times 10^{8}$ & $3.04 \times 10^{8}$ & $5.07 \times 10^{7}$ & $4.27 \times 10^{8}$ \\
\hline
\end{tabular}

${ }^{a}$ The zero of energy for the partition functions is the energy of the lowest-energy classical equilibrium structure.

The differences between methods are smaller for transition states, in part because the partition functions are smaller for the transition states. But the variation between reactions is still

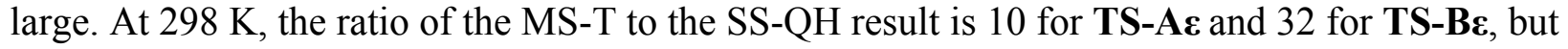
they are only 1.8 for TS-A $\boldsymbol{\alpha}$ and 2.3 for TS-B $\boldsymbol{\alpha}$. Thus the effect of the greater torsional phase space of the transition state for the $\varepsilon-\mathrm{H}$ shift as compared to the $\alpha-\mathrm{H}$ shift is a factor of $10 / 1.8=$ 5.6 for the competition of reactions of $\mathbf{A}$ and 32/2.3 $=14$ for the competition of reactions of $\mathbf{B}$. The effects increase at high temperature. By $1000 \mathrm{~K}$ the MS-T/SS-QH ratio has increased to 107.8 for TS-A $\boldsymbol{\varepsilon}$ and to 247.4 for TS-BE, while the ratios for TS-A $\boldsymbol{\alpha}$ and TS-B $\boldsymbol{\alpha}$ have increased

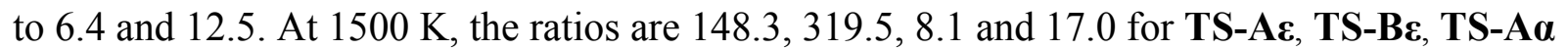
and TS-B $\boldsymbol{\alpha}$, respectively. These large effects have never before been directly calculated.

The multistructural torsional anharmonicity factor for every species ( $F_{\mathrm{X}}^{\mathrm{MS}-\mathrm{T}}$ in eq. 6$)$ and for every forward and reverse reaction $\left(F_{\mathrm{act}}^{\mathrm{MS}-\mathrm{T}}\right.$ in eq. 7$)$ is shown in Figure 3 for the temperature 
range of $298-1500 \mathrm{~K}$. The multistructural torsional anharmonicity factors $F_{\mathrm{X}}^{\mathrm{MS}-\mathrm{T}}$ for reactants A and B vary over the wide range of 217-3371 and 40-1049, respectively, but there is some cancellation of anharmonicity effects between reactants and transition states, so the reaction factors $F_{\mathrm{X}}^{\mathrm{MS}-\mathrm{T}}$ are spread over the narrower (but still huge) ranges of 1.8-8.1 and 2.3-17.0 for TS-A $\boldsymbol{\alpha}$ and TS-B $\boldsymbol{\alpha}$. We see the $F_{\mathrm{X}}^{\mathrm{MS}-\mathrm{T}}$ factor is much lower for TS-A $\boldsymbol{\alpha}$ than that for TS-A ; and as a consequence, according to eq 7 , for reaction $\mathrm{A} \alpha$, the multistructural torsional anharmonicity factor $\left(F_{\mathrm{act}}^{\mathrm{MS}-\mathrm{T}}\right)$ will be much lower than that for reaction A $\varepsilon$. Therefore the multistructural torsional anharmonicity will reduce the rate constants of A $\alpha$ to a larger extent than those for AE.

We also calculated the effects for the reverse reactions. As shown in Figure 3(ii), $F_{\text {act }}^{\mathrm{MS}-\mathrm{T}}$ is as low as 0.002 for the reverse reaction $A \alpha^{\prime}$ of $A \alpha$ in the temperature range of $1200-1500 \mathrm{~K}$. Figure 3 shows that the change of $F_{\text {act }}^{\mathrm{MS}-\mathrm{T}}$ is slightly smooth for reaction A $\varepsilon$, which decreases from 0.047 at $298 \mathrm{~K}$ to 0.044 at $1500 \mathrm{~K}$, but the ones for the reverse reactions ( $\mathrm{A} \varepsilon^{\prime}, \mathrm{A} \alpha^{\prime}, \mathrm{B} \varepsilon^{\prime}$, $\left.\mathrm{B} \alpha^{\prime}\right)$ are much steeper. In particular $F_{\mathrm{act}}^{\mathrm{MS}-\mathrm{T}}$ for reactions $\mathrm{A} \varepsilon^{\prime}$ and $\mathrm{A} \alpha^{\prime}$ decrease from 0.605 to 0.092 and from 0.653 to 0.004 over the studied temperature range. The ratio of $F_{\mathrm{act}}^{\mathrm{MS}-\mathrm{T}}$ for $\mathrm{A} \varepsilon$ to that for $A \alpha$ reflects the effect of multistructural torsional anharmonicity on the competing relationship for the forward hydrogen shift, which increases from a factor of 5.6 to a factor of 18.3 over the studied temperature range. However the ratio of $F_{\mathrm{act}}^{\mathrm{MS}-\mathrm{T}}$ for $\mathrm{B} \varepsilon$ to that for $\mathrm{B} \alpha$ has a nonmonotonic trend, e.g. it increases from a factor of 14.1 to 20.0 in the temperature range of $298-800 \mathrm{~K}$, but decreases from a factor of 20.0 to 18.8 in the temperature range of $800-1500$ $\mathrm{K}$. For comparison we note that in our previously studied OOQOOH system ${ }^{32}$, the ratio for $\varepsilon-\mathrm{H}$ shift to that for the $\alpha-H$ shift increases from a factor of 8 to a factor of 40 , so this effect should not be neglected at low temperature, and it becomes disastrous to neglect it at high temperature.

We conclude that the torsional anharmonicity plays a huge role on the competition between the two possible [1,5] hydrogen shifts, and the size of the effect depends on the structure of the alkyl group. 


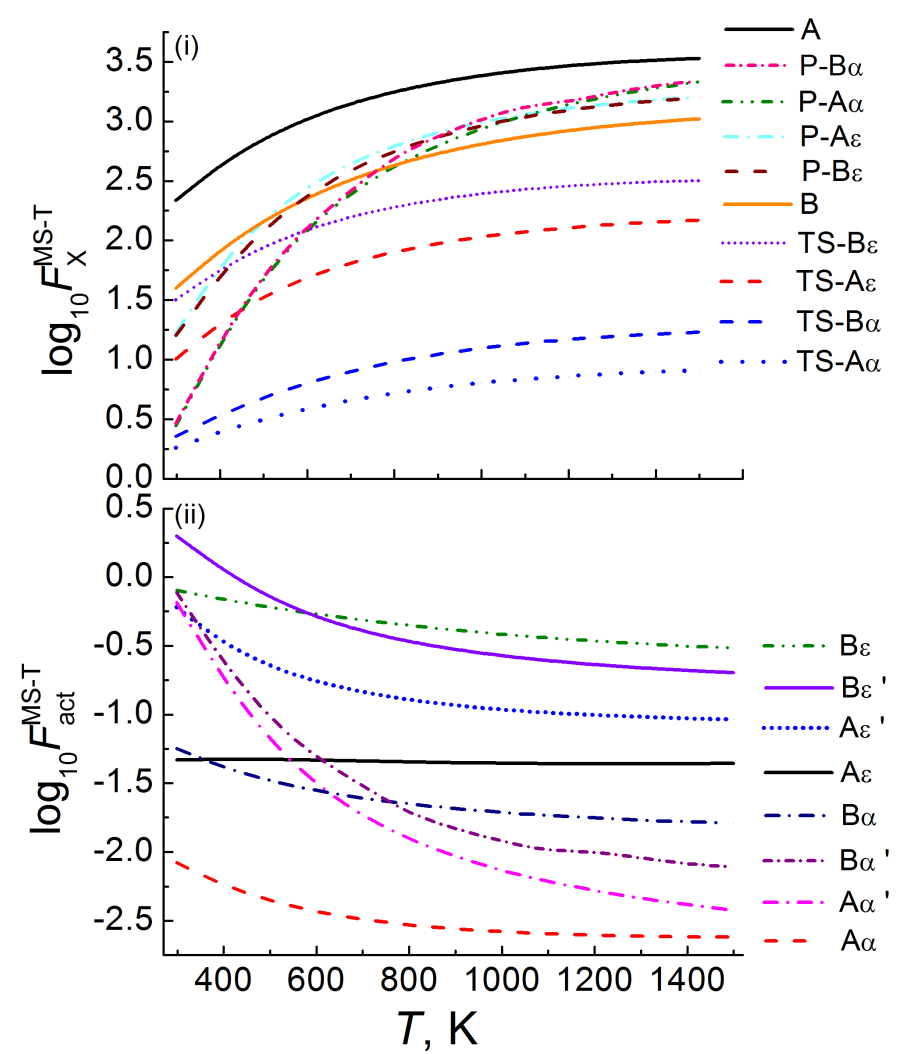

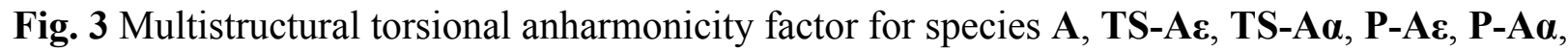
$\mathbf{B}, \mathbf{T S}-\mathbf{B} \boldsymbol{\varepsilon}, \mathbf{T S}-\mathbf{B} \boldsymbol{\alpha}, \mathbf{P}-\mathbf{B} \boldsymbol{\varepsilon}, \mathbf{P}-\mathbf{B} \boldsymbol{\alpha}$ and for each forward $(\mathrm{A} \varepsilon, \mathrm{A} \alpha, \mathrm{B} \varepsilon, \mathrm{B} \alpha)$ and reverse (A $\varepsilon^{\prime}, \mathrm{A} \alpha^{\prime}$, $\mathrm{B} \varepsilon^{\prime}, \mathrm{B} \alpha^{\prime}$ ) reaction.

\subsection{Thermodynamic properties}

All the thermodynamics quantities are determined by the total partition functions. The multistructural torsional anharmonicity plays a significant role in determining the conformational-rotational-vibrational partition functions, and therefore it directly affects the accuracy of these thermodynamics quantities. Table 3 gives the calculated temperaturedependent standard-state (one bar) ideal-gas entropy $S_{T}^{0}$, heat capacity $C_{P}^{0}(T)$, and relative enthalpy $H_{T}^{0}$ using the MS-T method both for reactants $\mathbf{A}$ and $\mathbf{B}$. For comparison, the thermodynamics quantities obtained by the MS-LQH and SS-QH approximations are also listed.

For $\mathbf{A}$, the multistructural torsional anharmonicity plays a significant role in the thermodynamic quantities, e.g. the deviation between MS-T and SS-QH is approximately 13.9-16.6 cal mol $\mathrm{c}^{-1} \mathrm{~K}^{-1}$ for entropy, 0.0-5.9 $\mathrm{cal} \mathrm{mol}^{-1} \mathrm{~K}^{-1}$ for heat capacity, and $0.1-2.5$ $\mathrm{kcal} / \mathrm{mol}$ for relative enthalpy. The deviation between MS-T and SS-QH for B is about 10.9-14.7 cal mol $\mathrm{K}^{-1}$ for entropy, 0.6-6.1 $\mathrm{cal} \mathrm{mol}^{-1} \mathrm{~K}^{-1}$ for heat capacity and $0.4-3.2 \mathrm{kcal} / \mathrm{mol}$ for relative enthalpy. The SS-QH approximation underestimates the entropy over the whole temperature range for $\mathbf{A}$ and $\mathbf{B}$. The deviation between the MS-T and SS-QH approximations has a non-monotonic variation, e.g., the deviation of entropy for $\mathbf{A}$ has a maximum of $16.6 \mathrm{cal} \mathrm{mol}^{-1}$ $\mathrm{K}^{-1}$ at $800 \mathrm{~K}$ and then it decreases with increasing temperature. The SS-QH approximation 
underestimates the heat capacity at low temperature, however it overestimates that at high temperature both for $\mathbf{A}$ and $\mathbf{B}$.

The MS-LQH results are much closer to the MS-T results than to the SS-QH results. This shows that the torsional anharmonicity is dominated more by the existence of multiple structures than by the torsional potential anharmonicity.

Table 3. Computed standard-state entropy $\left(\mathrm{cal} \mathrm{mol}^{-1} \mathrm{~K}^{-1}\right)$, heat capacity $\left(\mathrm{cal} \mathrm{mol}^{-1} \mathrm{~K}^{-1}\right)$, and relative enthalpy $(\mathrm{kcal} / \mathrm{mol})^{a}$ for reactants $\mathbf{A}$ and $\mathbf{B}$ using three methods

\begin{tabular}{|c|c|c|c|c|c|c|c|c|c|c|}
\hline & \multicolumn{4}{|c|}{$S_{T}^{0}$} & \multicolumn{3}{|c|}{$C_{P}^{0}(T)$} & \multicolumn{3}{|c|}{$H_{T}^{0}$} \\
\hline & $\mathrm{T}(\mathrm{K})$ & SS-QH ${ }^{b}$ & MS-LQH & MS-T & SS-QH ${ }^{b}$ & MS-LQH & MS-T & SS-QH ${ }^{b}$ & MS-LQH & MS-T \\
\hline \multirow[t]{9}{*}{$\mathrm{A}$} & 298 & 110.7 & 124.1 & 124.6 & 44.6 & 49.3 & 50.5 & 128.0 & 129.1 & 129.4 \\
\hline & 400 & 125.5 & 140.1 & 140.9 & 56.6 & 59.5 & 60.5 & 133.2 & 134.7 & 135.0 \\
\hline & 600 & 152.3 & 167.7 & 168.7 & 75.8 & 77.2 & 77.4 & 146.5 & 148.4 & 148.9 \\
\hline & 800 & 176.1 & 191.8 & 192.7 & 89.2 & 90.0 & 89.2 & 163.1 & 165.2 & 165.6 \\
\hline & 1000 & 197.1 & 212.9 & 213.6 & 98.9 & 99.3 & 97.6 & 182.0 & 184.2 & 184.3 \\
\hline & 1200 & 215.8 & 231.6 & 231.9 & 105.9 & 106.2 & 103.7 & 202.5 & 204.8 & 204.5 \\
\hline & 1500 & 240.3 & 256.2 & 255.8 & 113.4 & 113.5 & 110.1 & 235.4 & 237.8 & 236.6 \\
\hline & 1800 & 261.4 & 277.4 & 276.3 & 118.3 & 118.4 & 114.2 & 270.2 & 272.7 & 270.3 \\
\hline & 2000 & 274.0 & 290.0 & 288.4 & 120.7 & 120.8 & 116.2 & 294.2 & 296.6 & 293.4 \\
\hline \multirow[t]{9}{*}{$\bar{B}$} & 298 & 117.5 & 128.0 & 128.4 & 50.4 & 54.9 & 56.5 & 145.8 & 147.0 & 147.3 \\
\hline & 400 & 134.3 & 146.0 & 146.8 & 64.0 & 67.5 & 69.1 & 151.7 & 153.3 & 153.7 \\
\hline & 600 & 164.6 & 177.4 & 178.7 & 85.7 & 87.8 & 88.5 & 166.8 & 169.0 & 169.6 \\
\hline & 800 & 191.5 & 204.8 & 206.1 & 100.9 & 102.3 & 101.8 & 185.5 & 188.0 & 188.7 \\
\hline & 1000 & 215.2 & 228.8 & 229.9 & 111.9 & 112.8 & 111.3 & 206.9 & 209.6 & 210.1 \\
\hline & 1200 & 236.4 & 250.1 & 250.8 & 119.9 & 120.6 & 118.3 & 230.1 & 233.0 & 233.1 \\
\hline & 1500 & 264.1 & 277.9 & 278.1 & 128.4 & 128.8 & 125.5 & 267.4 & 270.5 & 269.7 \\
\hline & 1800 & 288.1 & 301.9 & 301.4 & 134.1 & 134.4 & 130.2 & 306.9 & 310.0 & 308.1 \\
\hline & 2000 & 302.3 & 316.2 & 315.2 & 136.8 & 137.0 & 132.4 & 334.0 & 337.2 & 334.4 \\
\hline
\end{tabular}

${ }^{a}$ The zero of energy is set equal to the potential energy of the global minimum.

${ }^{b}$ Calculated using the lowest-energy conformer.

\subsection{High-pressure-limit rate constants}

The high-pressure-limit rate constants by various methods are given in Figure 4. The final MS-CVT/SCT rate constants reflect the combined effects of multistructural torsional anharmonicity, the variational effects, and the multidimensional tunneling effects. The effect of multistructural torsional anharmonicity has already been discussed in subsection 3.2, and detailed information about the tunneling transmission coefficients and canonical recrossing transmission coefficients are also given in Figure 6 and Figure 7 respectively. 

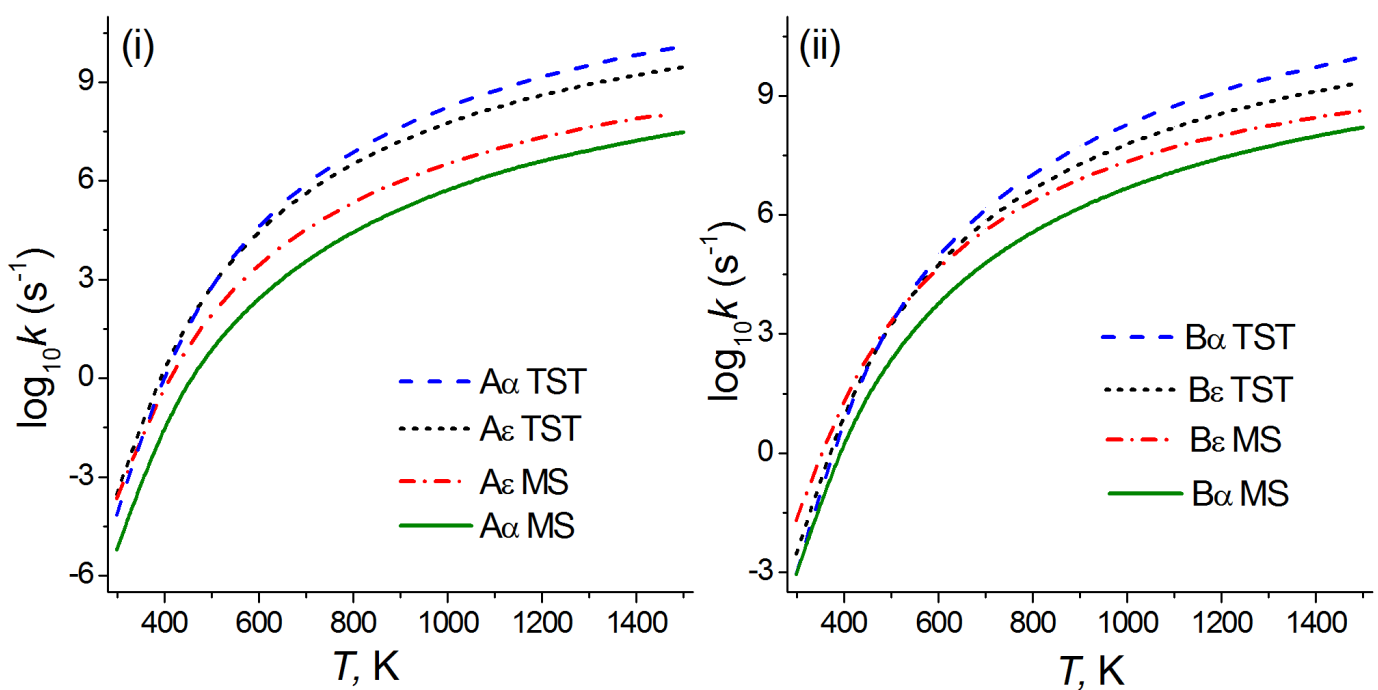

Fig. 4 High-pressure-limit rate constants by TST and MS-VTST/SCT for A $\varepsilon, A \alpha, B \varepsilon$ and $B \alpha$ at 298-1500 K. The MS-VTST/SCT results are labeled MS, and the single-structure conventional TST results without tunneling are labeled TST.

Figure 4 shows the common logarithms of rate constants for the forward reactions as calculated using both single-structure conventional TST and MS-CVT/SCT. We draw attention to five noteworthy phenomenon in Figure 4:

(i) For reaction $\mathrm{A} \varepsilon$, the final MS-CVT/SCT rate constants are differ from the singlestructure conventional transition state theory ones without tunneling by factors of 1.4-24. The small deviation at low temperatures originates from the compensation of the tunneling effect and multistructural torsional anharmonicity effect, since the former effect can increase the rate constants and the latter decreases them for the present reactions. As temperature increases, the deviation becomes larger, e.g. the MS-CVT/SCT rate constants differ from TST by factors of 18-24 at 1000-1500 K.

(ii) For reaction $\mathrm{B} \varepsilon$, the final MS-CVT/SCT rate constants exhibit a different trend as compared to the TST ones, e.g. at 298-500 K, the MS-CVT/SCT rate constants are slightly higher than those from TST; however when the temperature is raised, the MS-CVT/SCT rate constants become lower than that from TST by factors of 1.2-5.0.

(iii) The deviation between TST and MS-CVT/SCT for A $\alpha$ is much larger than that for the other reactions in the whole studied temperature range, which spread over a large range by factors of 12-415.

(iv) The different computational methods produce significantly different temperature dependences, e.g., the final calculations of $\mathrm{A} \alpha$ and $\mathrm{B} \alpha$ have a weaker positive temperature dependence than is calculated by TST. This is seen more quantitatively by comparing Arrhenius activation energies; the Arrhenius activation energy at temperature $T$ is defined as

$$
E_{\mathrm{a}}=-d \ln k(\mathrm{~T}) / d(1 / T),
$$

and these values are shown in Figure 5. 


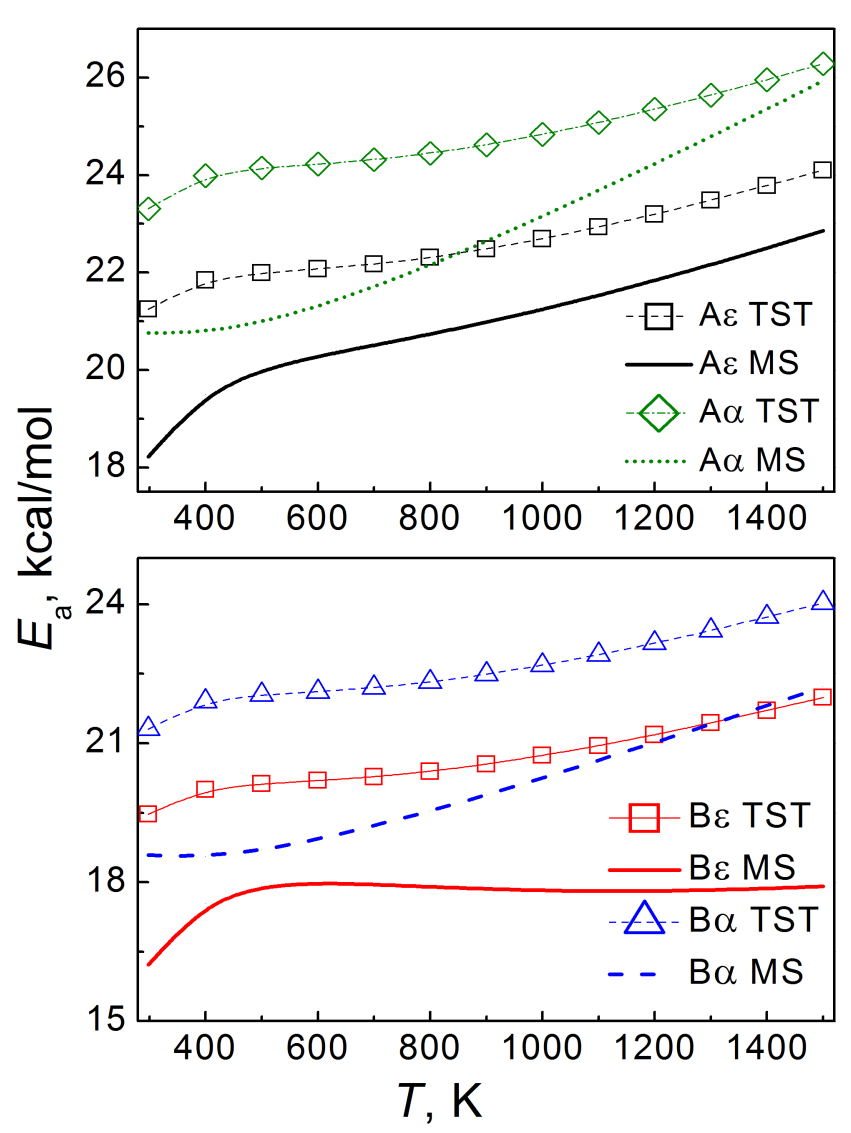

Figure 5. High-pressure-limit activation energies by different methods.

(v) The MS-CVT/SCT method predicts that reaction A $\varepsilon$ always dominates over A $\alpha$ for the whole studied temperature range; however in the temperature range of 500-1500 K, the rate constants of A $\alpha$ are higher than those of A $\varepsilon$ calculated by TST method. This can be explained by a combination of three factors: firstly, the result originates from the influence of the multistructural torsional anharmonicity as can be seen in Figure 3, e.g., the multistructural torsional anharmonicity factors for A $\varepsilon$ are $0.047,0.047$ and 0.044 at 298, 600 and $1000 \mathrm{~K}$, respectively, but for A $\alpha$ they are 0.008, 0.004 and 0.003, respectively, and these smaller multistructural torsional anharmonicity factors for A $\alpha$ decrease the rate constants of $A \alpha$ dramatically in the whole studied temperature range. Secondly, the tunneling transmission coefficients (SCT) in Figure 6 for A $\varepsilon$ are 15.8, 2.14, and 1.54, at 298, 600 and $800 \mathrm{~K}$, respectively, but for A $\alpha$ they are 10.6, 1.76, and 1.30, respectively, and these slightly higher recrossing transmission coefficients for $A \varepsilon$ contribute to the higher rate constants of $A \varepsilon$ at low temperatures. Lastly, the canonical recrossing transmission coefficient for A $\varepsilon$ is larger than those of $A \alpha$, as can be seen in Figure 7.

Figure 6 shows the ZCT and SCT transmission coefficients as functions of temperature. We see that ZCT significantly underestimate the tunneling at $298 \mathrm{~K}$ and the deviation between the ZCT and SCT approximations decreases with increasing temperature. At $298 \mathrm{~K}$, the ZCT transmission coefficient is equal to 5.8 and 4.0 for reactions $\mathrm{A} \varepsilon$ and $\mathrm{A} \alpha$; the corresponding SCT 
transmission coefficients are 15.8 and 10.7 respectively. Tunneling increases the reaction rate constants to a different extent for different reactions; in the temperature range of 298-800 K, the SCT transmission coefficients cover the range of 1.5-15.8 for A $\varepsilon, 1.3-10.7$ for A $\alpha, 1.3-9.9$ for $\mathrm{B} \varepsilon$, and 1.6-17.8 for $\mathrm{B} \alpha$. Previous studies for hydrogen shift reaction ${ }^{3,4}$ have shown that the SCT approximation is much more reliable than the Wigner method ${ }^{75}$ and the ZCT approximation. Thus we adopt the SCT results for our final values and for the following falloff calculations.

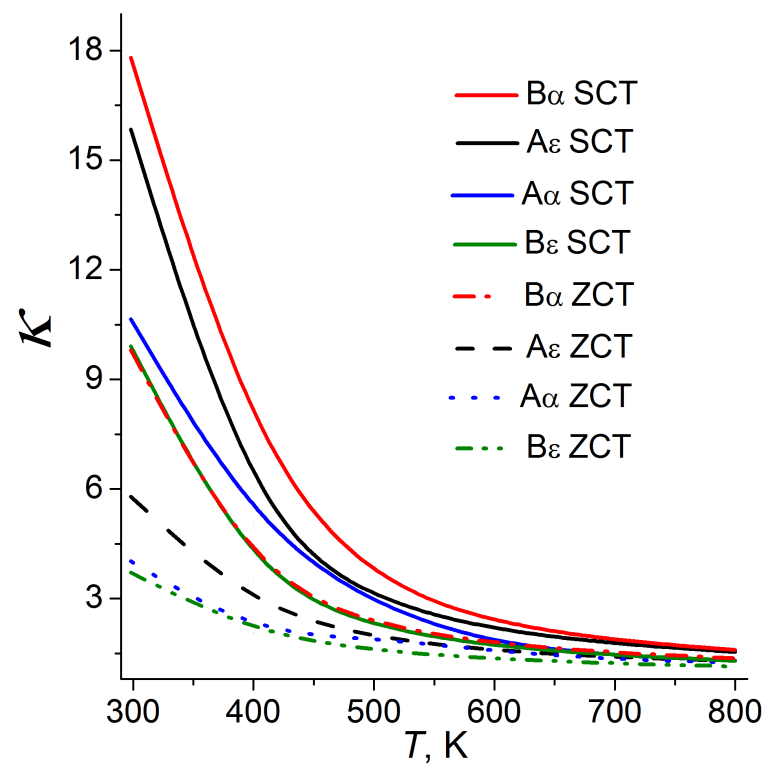

Fig. 6 Tunneling transmission coefficients by small-curvature tunneling (SCT) and zerocurvature tunneling (ZCT) method for $\mathrm{A} \varepsilon, \mathrm{A} \alpha, \mathrm{B} \varepsilon$ and $\mathrm{B} \alpha$ at $298-800 \mathrm{~K}$.

In the present work, the canonical recrossing transmission coefficient is defined as the ratio of rate constants computed by quasiclassical variational TST to that computed by quasiclassical conventional TST. Figure 7 shows the CVT canonical recrossing transmission coefficients (labeled as $\Gamma^{\mathrm{CVT}}$ ) for reactions $\mathrm{A} \varepsilon, \mathrm{A} \alpha, \mathrm{B} \varepsilon$ and $\mathrm{B} \alpha$. It can be seen that the factors are in the range of $0.70-0.99$ over the temperature interval $298-1500 \mathrm{~K}$. Thus the present work indicates that the variational effect is small. 


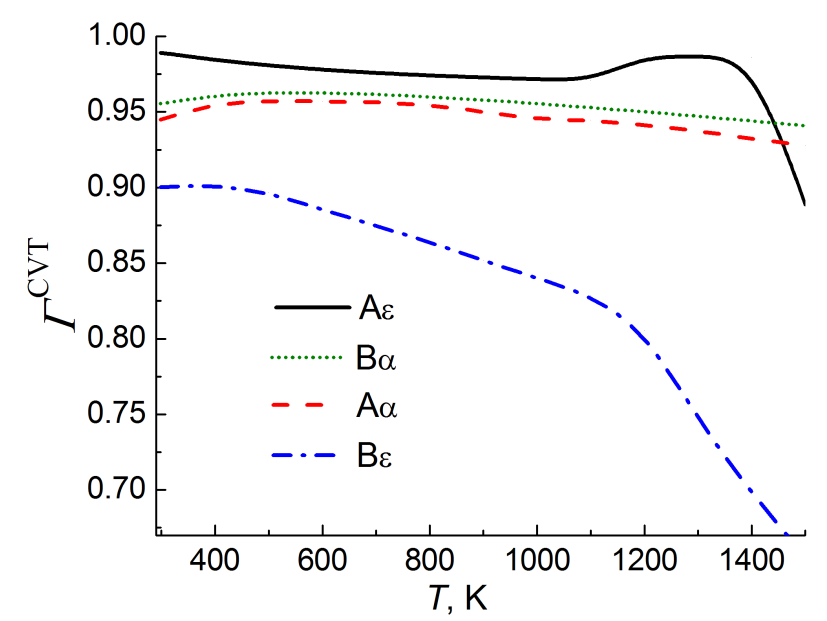

Fig. 7 Canonical variational recrossing coefficients $\left(\Gamma^{\mathrm{CVT}}\right)$ for $\mathrm{A} \varepsilon, \mathrm{A} \alpha, \mathrm{B} \varepsilon$ and $\mathrm{B} \alpha$.

Having reviewed the individual contributions to the MS-VTST/SCT rate constants, we conclude that the multiple structure anharmonicity is the most important correction to conventional transition state theory based on the lowest-energy structures for reactants and transition states. To isolate this effect, Table 4 shows comparisons of high-pressure-limit rate constants for forward reactions by MS-VTST/SCT and by SS-VTST/SCT; the difference is entirely due to multistructural torsional anharmonicity. Over the temperature range 298-1500 K, the final MS-VTST/SCT rate constants for all four reactions are always smaller than the singlestructure canonical variational TST results (including tunneling), but the size of the decrease depends on the reaction. This is illustrated in Table 5, which shows that the decrease is a factor of 21-23 for reaction $A \varepsilon$, a factor of 120-417 for reaction $A \alpha$, a factor of 1.2-3.3 for reaction $\mathrm{B} \varepsilon$, and a factor of 18-62 for reaction $\mathrm{B} \varepsilon$; within these ranges, the factor becomes larger as temperature increases. In the $800-1100 \mathrm{~K}$ range the factors vary from 2.2 to 23 for $\varepsilon-\mathrm{H}$ shifts and from 45 to 393 for $\alpha-H$ shifts.

Table 4. Comparisons of the high-pressure-limit rate constants for forward reactions by MSVTST/SCT and SS-VTST /SCT. ${ }^{a}$

\begin{tabular}{ccccccccc}
\hline & \multicolumn{2}{c}{$\mathrm{A} \varepsilon$} & \multicolumn{2}{c}{$\mathrm{A} \alpha$} & \multicolumn{2}{c}{$\mathrm{B} \varepsilon$} & \multicolumn{2}{c}{$\mathrm{B} \alpha$} \\
\hline$T(\mathrm{~K})$ & $\mathrm{MS}$ & $\mathrm{SS}$ & $\mathrm{MS}$ & $\mathrm{SS}$ & $\mathrm{MS}$ & $\mathrm{SS}$ & $\mathrm{MS}$ & $\mathrm{SS}$ \\
\hline 298 & $2.20 \times 10^{-4}$ & $4.72 \times 10^{-3}$ & $6.09 \times 10^{-6}$ & $7.28 \times 10^{-4}$ & $2.06 \times 10^{-2}$ & $2.57 \times 10^{-2}$ & $9.09 \times 10^{-4}$ & $1.60 \times 10^{-2}$ \\
500 & $1.11 \times 10^{2}$ & $2.35 \times 10^{3}$ & $9.80 \times 10^{0}$ & $2.23 \times 10^{3}$ & $2.67 \times 10^{3}$ & $4.43 \times 10^{3}$ & $2.88 \times 10^{2}$ & $8.78 \times 10^{3}$ \\
800 & $2.36 \times 10^{5}$ & $5.23 \times 10^{6}$ & $2.93 \times 10^{4}$ & $9.97 \times 10^{6}$ & $2.34 \times 10^{6}$ & $5.26 \times 10^{6}$ & $3.93 \times 10^{5}$ & $1.76 \times 10^{7}$ \\
1100 & $9.13 \times 10^{6}$ & $2.08 \times 10^{8}$ & $1.65 \times 10^{6}$ & $6.49 \times 10^{8}$ & $5.31 \times 10^{7}$ & $1.47 \times 10^{8}$ & $1.25 \times 10^{7}$ & $6.78 \times 10^{8}$ \\
1500 & $1.18 \times 10^{8}$ & $2.69 \times 10^{9}$ & $3.00 \times 10^{7}$ & $1.25 \times 10^{10}$ & $4.33 \times 10^{8}$ & $1.42 \times 10^{9}$ & $1.60 \times 10^{8}$ & $9.89 \times 10^{9}$ \\
\hline
\end{tabular}

${ }^{a}$ The MS-VTST/SCT results are labeled MS; the SS-VTST/SCT ones are labeled SS.

The last two columns of Table 5 compare the multiple-structure effects on the rate constants for the same reaction ( $\varepsilon-\mathrm{H}$ shift or $\alpha-\mathrm{H}$ shift) but with different alkyl groups. We see that not only do the multiple-structure effects have large effects on reaction rates, they also have large effects 
on ratios of reaction rates, even for the seemingly minor change of replacing a 1-hexyl group (in A) by a 5-methyl-1-hexyl group (in B). Thus, even though there is some cancellation between the anharmonicity of the reactant and the anharmonicity of the transition state, and even though the reactants are very similar in structure, differing only by a methyl group, the effect of multiple structure anharmonicity has a large effect on the relative rates -as large as a factor of 17 at room temperature and as large as a factor of 7 at $1500 \mathrm{~K}$.

Table 5. Ratios of the high-pressure-limit rate constants for forward reactions by SS-VTST/SCT and MS-VTST /SCT. ${ }^{a}$

\begin{tabular}{|c|c|c|c|c|c|c|}
\hline \multirow[b]{2}{*}{$T(\mathrm{~K})$} & \multicolumn{2}{|c|}{$\mathrm{A} \alpha$} & \multicolumn{2}{|c|}{$\mathrm{B} \alpha$} & \multicolumn{2}{|c|}{$\mathrm{SS} / \mathrm{MS}$} \\
\hline & $\mathrm{SS} / \mathrm{MS}$ & SS/MS & $\mathrm{SS} / \mathrm{MS}$ & $\mathrm{SS} / \mathrm{MS}$ & $\mathrm{A} \varepsilon / \mathrm{B} \varepsilon$ & $\mathrm{A} \alpha / \mathrm{B} \alpha$ \\
\hline 298 & 21 & 120 & 1.2 & 18 & 17.2 & 6.8 \\
\hline 500 & 21 & 228 & 1.7 & 30 & 12.8 & 7.5 \\
\hline 800 & 22 & 340 & 2.2 & 45 & 9.9 & 7.6 \\
\hline 1100 & 23 & 393 & 2.8 & 54 & 8.2 & 7.3 \\
\hline 1500 & 23 & 417 & 3.3 & 62 & 7.0 & 6.7 \\
\hline
\end{tabular}

${ }^{a}$ The MS-VTST/SCT results are labeled MS; the SS-VTST/SCT ones are labeled SS.

Table 6 gives fits to our computed rate constants for all four reactions in the high-pressure limit. (The rate constants at other pressures may be useful for modeling alkane oxidation and are also of fundamental interest for chemical kinetics, and they are provided in the Supporting Information and discussed in the next subsection.) Because the reactions are endothermic we fit the rate constants to the following form: ${ }^{76}$

$$
k(T)=A\left(\frac{T}{300}\right)^{n} \exp \left[-\frac{E\left(T+T_{0}\right)}{R\left(T^{2}+T_{0}^{2}\right)}\right]
$$

where $A, n, E$ and $T_{0}$ are parameters.

Table 6. Fitting parameters for the high-pressure-limit forward MS-CVT/SCT rate constants

\begin{tabular}{ccccc}
\hline & $\begin{array}{c}A \\
\left(\mathrm{~s}^{-1}\right)\end{array}$ & $\mathrm{n}$ & $\begin{array}{c}T_{0} \\
(\mathrm{~K})\end{array}$ & $\begin{array}{c}E \\
(\mathrm{kcal} / \mathrm{mol})\end{array}$ \\
\hline $\mathrm{A} \varepsilon$ & $4.49 \times 10^{8}$ & 2.36 & 121.68 & 13.88 \\
$\mathrm{~A} \alpha$ & $4.49 \times 10^{7}$ & 3.15 & 37.08 & 15.80 \\
$\mathrm{~B} \varepsilon$ & $1.55 \times 10^{10}$ & 0.82 & 134.39 & 13.42 \\
$\mathrm{~B} \alpha$ & $8.32 \times 10^{8}$ & 2.21 & 23.83 & 15.17 \\
\hline
\end{tabular}

\subsection{High-pressure branching fractions}

The branching fractions for [1,5] hydrogen shifts of OOQOOH species are not available experimentally due to the reactivity of OOQOOH species; thus our calculations in the present study can be especially useful in guiding combustion mechanisms. The branching fraction is defined here as the ratio of the rate constant for the $\varepsilon-\mathrm{H}$ shift to the sum of the rate constants for the $\alpha-\mathrm{H}$ shift and the $\varepsilon-\mathrm{H}$ shift. The branching fractions are plotted in Figure 8: the solid curves 
are MS-CVT/SCT results in the high-pressure limit; the dashed curves are predictions by conventional TST without tunneling. The figure also shows the branching fraction from our previous OOQOOH paper. ${ }^{32}$. For reactants A and B, the MS-CVT/SCT give very similar results, with the $\varepsilon-\mathrm{H}$ shift dominating over the whole temperature range (the branching fraction exceeds 0.5 ), but TST gives a much smaller branching fraction. Thus it is risky to use the conventional single-structure harmonic transition state theory (TST).

For reactant $\mathbf{C}$, the results are quite different than those for $\mathbf{A}$ and $\mathbf{B}$. As mentioned in the introduction, the $\alpha-\mathrm{H}$ position is always at secondary carbons for these three studied case (A, B, and $\mathbf{C}$ ), but the hydrogen atom transferred in the $\varepsilon-\mathrm{H}$ shift is at secondary carbon for reactant $\mathbf{A}$, at a tertiary carbon for reactant $\mathbf{B}$, and at a primary carbon for reactant $\mathbf{C}$. The much smaller branching fractions for reaction $\mathrm{C} \varepsilon$ are consistent with the higher bond energy of primary $\mathrm{C}-\mathrm{H}$ bonds.

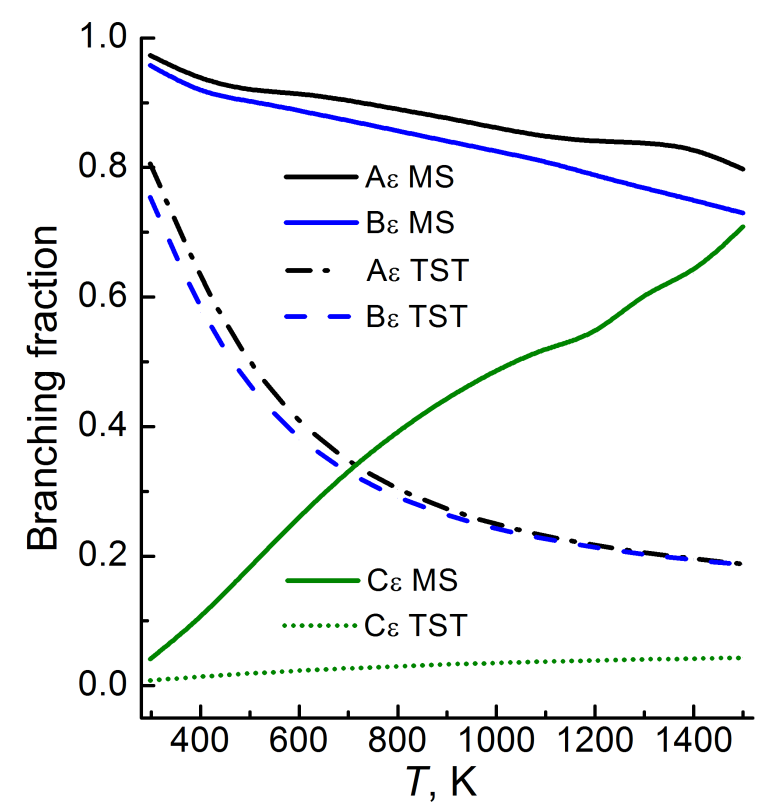

Fig. 8 Branching fraction of reactions $A \varepsilon, B \varepsilon$, and $C \varepsilon$ as functions of temperature as predicted by MS-CVT/SCT high-pressure limit rate constants (solid lines) and by TST method (dashed lines). The branching fraction is defined here as the ratio of the rate constant for the $\varepsilon-\mathrm{H}$ shift to the sum of the rate constants for the $\alpha-\mathrm{H}$ shift and the $\varepsilon-\mathrm{H}$ shift. Here $\mathrm{C} \varepsilon$ represents the same type reaction in the series of our previous OOQOOH paper. ${ }^{32}$

\subsection{Pressure-dependent rate constants and branching fractions}

The treatment of reaction rates used here makes the independent-reaction assumption that assumes that each reaction may be treated individually. The independent-reaction assumption is entirely correct for all the results discussed so far, which correspond to the high-pressure limit, but it is an extra assumption for calculating falloff effects because the pressure dependence of a given reaction can be strongly influenced by the reactions that compete with it. Therefore the rate constants must eventually be studied in the context of an overall reaction mechanism modeled by a master equation. ${ }^{77-80}$ For the case of competing isomerization reactions, each competing reaction depletes the same reservoir of reactive states of a given reactant, and this lowers the 
reaction rates as compared to those calculated by the independent-reaction assumption. ${ }^{81}$ Since the effect depends on the relative threshold energies of the competing reactions, it can also affect the pressure-dependent branching ratios. ${ }^{81}$ Nevertheless it is interesting to consider the independent-reaction falloff effects for the isomerizations because they are independent of mechanism and can be considered as well-defined lower bounds on the competing-reaction pressure-dependent falloff effects (i.e., as giving approximate upper bounds on the pressuredependent rate constants). Because the independent-reaction assumption becomes more severe at low pressure, ${ }^{81}$ we will only discuss the pressure dependence for pressures of $1 \mathrm{~atm}$ and above.

Figure 9 shows the predicted falloff results obtained by the independent-reaction SS-QRRK theory. For pressures greater than $1 \mathrm{~atm}$, there is negligible pressure dependence for temperatures below about $500 \mathrm{~K}$ for reactions of $\mathbf{A}$ and $\mathbf{B}$. As the temperature increases, the rate constants at low pressure differ more and more from the high-pressure limits, and for temperatures above $1100 \mathrm{~K}$, the difference between the rate constants at $100 \mathrm{~atm}$ and those in the high-pressure limit are factors of $7.2-1.3 \times 10^{6}$ for $A \varepsilon, 7.6-1.4 \times 10^{6}$ for $A \alpha$, and up to nine orders of magnitude for $B \varepsilon$ and $\mathrm{B} \alpha$; thus the falloff effect cannot be neglected even in high-pressure engines. At atmospheric pressure (1 atm), the rate constants differ from those in the high-pressure limit by factors of 9.5-9684 for $\mathrm{A} \varepsilon, 10.1-10366$ for $\mathrm{A} \alpha, 44.7-1.2 \times 10^{6}$ for $\mathrm{B} \varepsilon, 48.5-1.3 \times 10^{6}$ for $\mathrm{B} \alpha$ in the critical temperature range $900-1200 \mathrm{~K}$. Figure 9 also shows that at low pressures and high temperatures, the independent-reaction rate constants have a negative temperature dependence. Figure S2 in the SI shows the simultaneous dependence of the independent-reaction branching fractions on temperature and pressure.

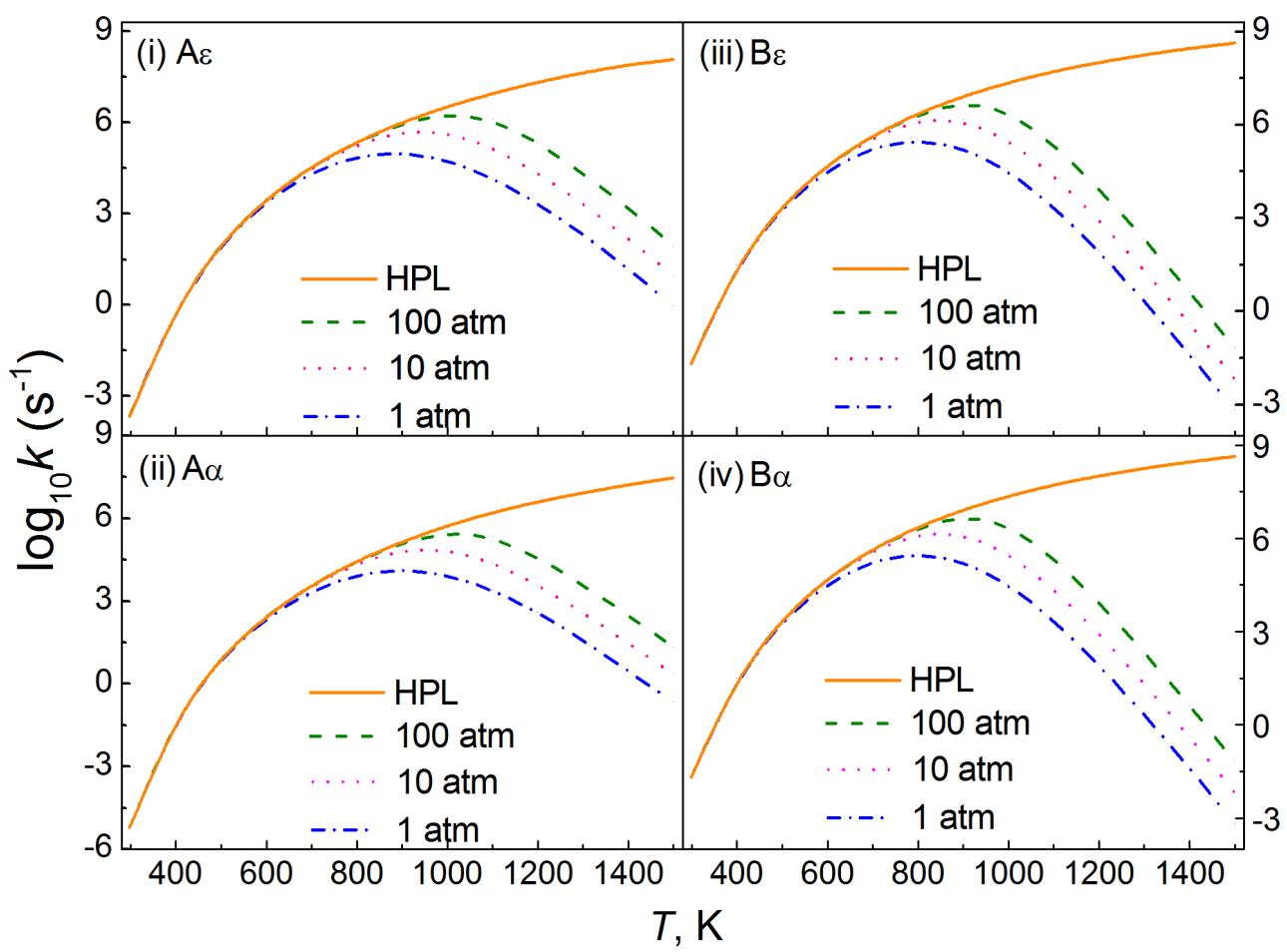

Fig. 9 Rate constant of $\mathrm{A} \varepsilon, \mathrm{A} \alpha, \mathrm{B} \varepsilon$ and $\mathrm{B} \alpha$ as functions of temperature at various pressures. 
We examined the sensitivity of the calculated pressure-dependent rate constants for reaction A $\varepsilon$ to the parameter $\Theta$ in the $\langle\Delta E\rangle_{\text {down }}$ formula by varying $\Theta$, and the results of these checks are provided in the SI. We find, as expected, that the sensitivity is much greater at high temperature. At $10 \mathrm{~atm}$ pressure, the difference between the results for $\Theta=300 \mathrm{~cm}^{-1}$ and $\Theta=900 \mathrm{~cm}^{-1}$ is a factor of 5.6 at $1000 \mathrm{~K}$, and a factor of 9.0 at $1500 \mathrm{~K}$.

\subsection{Implication for OOQOOH chemistry}

It is essentially impossible to detect the $\mathrm{H}$-shift reactions experimentally due to the high reactivity of OOQOOH species. Therefore, theoretical calculations are essential for understanding the kinetics of these $\mathrm{H}$-shift reactions. However, competition among the H-shift reactions at different sites of OOQOOH lead to a particular distribution of final products (P-A $\boldsymbol{\alpha}$, $\mathbf{P}-\mathbf{B} \boldsymbol{\alpha} \ldots$..., which are not distinguished by current experimental diagnostics. It is especially important to note that accurate reverse reactions are essential for the combustion models. The thermodynamics and kinetics data computed by high-level calculations in this work can lead to improved accuracy in chemical kinetics models of alkane autoxidation for both forward and reverse reactions. In selecting a density functional for direct dynamics, we usually look not only at the forward barrier height but also at the reverse barrier height. This is motivated primarily by the fact that the location of the transition state (which has a controlling effect on its vibrational frequencies and hence on its partition function) is usually sensitive to the calculated energy of reaction, so we want get that right, but a side effect is that our predictions of the backward reaction rates are also more accurate.

It is worth noting the subsequent fate of these dihydroperoxyalkyl species for the overall oxidation mechanism, even though it is out of the scope of this study. The weak $\mathrm{O}-\mathrm{OH}$ bond in the dihydroperoxyalkyl species (e.g. P-A $\boldsymbol{\alpha}$ and $\mathbf{P}-\mathbf{B} \boldsymbol{\alpha}$ ) then breaks to form $\mathrm{OH}$ and a ketohydroperoxide. This sequence of chain branching reactions is the reason for low-temperature reactivity leading to chemical and thermal runaway. The dihydroperoxyalkyl species (e.g. P-AE and $\mathbf{P}-\mathbf{B} \boldsymbol{\varepsilon}$ ) through $\boldsymbol{\varepsilon}-\mathrm{H}$ isomerization can undergo extensive autooxidation (third $\mathrm{O}_{2}$ addition mechanism) leading to highly oxygenated intermediates. ${ }^{28,31,82}$ The competition between $\varepsilon-\mathrm{H}$ isomerization reactions and $\alpha-\mathrm{H}$ isomerization determines in present study that molecules like $\mathbf{A}$ and $\mathbf{B}$ have a great trend to undergo extensive autooxidation leading to highly oxygenated intermediates.

\section{CONCLUDING REMARKS}

Multistructural canonical variational transition-state-theory with multidimensional tunneling has been used to calculate high-pressure limiting rate constants for internal [1,5] hydrogen shifts of typical hydroperoxyalkylperoxy $(\mathrm{OOQOOH})$ radicals in the kinetics of the second oxidation mechanism of alkane combustion, and the SS-QRRK method was used to estimate the pressure dependence. Our calculations include accurate determinations of the classical barrier heights, the variational effects, internal-coordinate-based vibrationally adiabatic potential curves, multistructural torsional anharmonicity, and multidimensional tunneling effects as put together by the multistructural VTST method. 
We found that multistructural torsional anharmonicity has a decisive effect on the thermodynamic data, on the forward and reverse reaction rates, and on the structural dependence of the reaction rates and branching fractions. The size of the effect depends strongly on the structure of the reactant. For example, it reduces the rate constants by factors of 0.044 to 0.047 for the $\varepsilon-\mathrm{H}$ shift $\mathrm{A} \varepsilon$, and it decreases the rate constants by factors of 0.002 to 0.008 for the $\alpha-\mathrm{H}$ shift $A \alpha$. It reduces the rate constants by factors of 0.09 to 0.6 for the reverse reaction $A \varepsilon^{\prime}$. Many rate constant estimations in combustion chemistry, atmospheric chemistry, and biological chemistry are estimated based mainly on bond energies, with no explicit consideration of this kind of entropic effect, and the present studies provide a caution that this may lead to large errors.

The optimization of the location of the variational transition state plays a minor role in the determination of the rate constants, but the tunneling effect is large at low temperatures, e.g., at $298 \mathrm{~K}$, the tunneling transmission coefficient is 15.8 for reaction $\mathrm{A} \varepsilon$ and 9.9 for reaction $\mathrm{B} \varepsilon$.

We found that the internal [1,5] hydrogen shift reactions of OOQOOH species are pressuredependent in the temperature range 700-1500 K, and the falloff effect cannot be ignored especially at the high end of the temperature range. It is worth noting that, contrary to a common assumption, the $\alpha-\mathrm{H}$ isomerizations $\mathrm{A} \alpha$ and $\mathrm{B} \alpha$ are the minor reactions over the investigated temperature range, and reactions $\mathrm{A} \varepsilon$ and $\mathrm{B} \varepsilon$ always dominate, which will have a significant effect on auto-oxidation mechanisms. The thermodynamic and kinetics data computed in this work should lead to improved accuracy in chemical kinetics models of alkane autoxidation.

\title{
ASSOCIATED CONTENT
}

\section{Supporting Information}

The Supporting Information is available free of charge on the ACS Publications website at DOI: $10.1021 /$ jacs

Cartesian coordinates, absolute energies, internal coordinates used for vibrational energies of generalized transition states, sensitivity checks for parameter $\Theta$ of $\langle\Delta E\rangle_{\text {down, }}$ and final calculated rate constants.

\author{
AUTHOR INFORMATION \\ ${ }^{\dagger}$ These authors contributed equally.

\section{Corresponding Authors} \\ *xing112018@haust.edu.cn \\ *truhlar@umn.edu

\section{ORCID} \\ Lili Xing: 0000-0003-2099-8472 \\ Junwei Lucas Bao: 0000-0002-4967-663X \\ Donald G. Truhlar: 0000-0002-7742-7294
}

\section{Notes}

The authors declare no competing financial interest. 


\section{ACKNOWLEDGMENTS}

This work was supported in part by Key Science Foundation of Higher Education of Henan (19A480002), by the U.S. Department of Energy, Office of Basic Energy Sciences, under Award Number DE-SC0015997. The authors are grateful to Feng Zhang for helpful discussions.

\section{REFERENCES}

(1) Eyring, H.; Lin, S. H.; Lin, S. M. Basic Chemical Kinetics; Wiley: New York, 1980.

(2) Yu, T.; Zheng, J.; Truhlar, D. G. Multi-structural variational transition state theory. Kinetics of the 1,4-hydrogen shift isomerization of the pentyl radical with torsional anharmonicity. Chem. Sci. 2011, 2, 2199-2213.

(3) Yu, T.; Zheng, J.; Truhlar, D. G. Multipath variational transition state theory: Rate constant of the 1,4-hydrogen shift isomerization of the 2-cyclohexylethyl radical. J. Phys. Chem. A 2012, 116, 297308.

(4) Xu, X.; Papajak, E.; Zheng, J.; Truhlar, D. G. Multi-structural variational transition state theory: kinetics of the 1,5-hydrogen shift isomerization of the 1-butoxyl radical including all structures and torsional anharmonicity. Phys. Chem. Chem. Phys. 2012, 14, 4204-4216.

(5) Alecu, I. M.; Zheng, J.; Papajak, E.; Yu, T.; Truhlar, D. G. Biofuel combustion. Energetics and kinetics of hydrogen abstraction from carbon-1 in n-butanol by the hydroperoxyl radical calculated by coupled cluster and density functional theories and multistructural variational transition-state theory with multidimensional tunneling. J. Phys. Chem. A 2012, 116, 12206-12213.

(6) Zheng, J.; Seal, P.; Truhlar, D. G. Role of conformational structures and torsional anharmonicity in controlling chemical reaction rates and relative yields: butanal + $\mathrm{HO} 2$ reactions. Chem. Sci. 2013, 4 (1), 200-212.

(7) Buda, F.; Bounaceur, R.; Warth, V.; Glaude, P. A.; Fournet, R.; Battin-Leclerc, F. Progress toward a unified detailed kinetic model for the autoignition of alkanes from C4 to C10 between 600 and $1200 \mathrm{~K}$. Combust. Flame 2005, 142, 170-186.

(8) Glowacki, D. R.; Pilling, M. J. Unimolecular reactions of peroxy radicals in atmospheric chemistry and combustion. ChemPhysChem 2010, 11, 3836-3843.

(9) Westbrook, C. K. Chemical kinetics of hydrocarbon ignition in practical combustion systems. Proc. Combust. Inst. 2000, 28, 1563-1577.

(10) Battin-Leclerc, F. Detailed chemical kinetic models for the low-temperature combustion of hydrocarbons with application to gasoline and diesel fuel surrogates. Prog. Energy Combust. Sci. 2008, 34, 440-498.

(11) Zádor, J.; Taatjes, C. A.; Fernandes, R. X. Kinetics of elementary reactions in low-temperature autoignition chemistry. Prog. Energy Combust. Sci. 2011, 37, 371-421.

(12) Battin-Leclerc, F. What do we really know about the low-temperature oxidation of alkanes. Proceedings of the European Combustion Meeting 2015, 1, 1-6.

(13) DeSain, J. D.; Taatjes, C. A.; Miller, J. A.; Klippenstein, S. J.; Hahn, D. K. Infrared frequencymodulation probing of product formation in alkyl +02 reactions. Part IV. Reactions of propyl and butyl radicals with 02. Faraday Discuss. 2002, 119, 101-120.

(14) DeSain, J. D.; Klippenstein, S. J.; Miller, J. A.; Taatjes, C. A. Measurements, theory, and modeling of $\mathrm{OH}$ formation in ethyl $+\mathrm{O}_{2}$ and propyl $+\mathrm{O}_{2}$ reactions. J. Phys. Chem. A 2003, 107, 44154427.

(15) Fernandes, R. X.; Zador, J.; Jusinski, L. E.; Miller, J. A.; Taatjes, C. A. Formally direct pathways and low-temperature chain branching in hydrocarbon autoignition: the cyclohexyl $+\mathrm{O}_{2}$ reaction at high pressure. Phys. Chem. Chem. Phys. 2009, 11, 1320-1327. 
(16) Battin-Leclerc, F.; Herbinet, O.; Glaude, P.-A.; Fournet, R.; Zhou, Z.; Deng, L.; Guo, H.; Xie, M.; Qi, F. Experimental confirmation of the low-temperature oxidation scheme of alkanes. Angew. Chem. Int. Ed. 2010, 49, 3169-3172.

(17) Battin-Leclerc, F.; Herbinet, O.; Glaude, P.-A.; Fournet, R.; Zhou, Z.; Deng, L.; Guo, H.; Xie, M.; Qi, F. New experimental evidences about the formation and consumption of ketohydroperoxides. Proc. Combust. Inst. 2011, 33, 325-331.

(18) Herbinet, O.; Husson, B.; Serinyel, Z.; Cord, M.; Warth, V.; Fournet, R.; Glaude, P.-A.; Sirjean, B.; Battin-Leclerc, F.; Wang, Z.; et al. Experimental and modeling investigation of the low-temperature oxidation of n-heptane. Combust. Flame 2012, 159, 3455-3471.

(19) Rotavera, B.; Zádor, J.; Welz, O.; Sheps, L.; Scheer, A. M.; Savee, J. D.; Akbar Ali, M.; Lee, T. S.; Simmons, B. A.; Osborn, D. L.; et al.. Photoionization mass spectrometric measurements of initial reaction pathways in low-temperature oxidation of 2,5-dimethylhexane. J. Phys. Chem. A 2014, 118, 10188-10200.

(20) Herbinet, O.; Battin-Leclerc, F. Progress in understanding low-temperature organic compound oxidation using a jet-stirred reactor. Int. J. Chem. Kinet. 2014, 46, 619-639.

(21) Wang, Z.; Herbinet, O.; Cheng, Z.; Husson, B.; Fournet, R.; Qi, F.; Battin-Leclerc, F. Experimental investigation of the low-temperature oxidation of the five isomers of hexane. J. Phys. Chem. A 2014, 118, 5573-5594.

(22) Eskola, A. J.; Welz, O.; Zádor, J.; Antonov, I. O.; Sheps, L.; Savee, J. D.; Osborn, D. L.; Taatjes, C. A. Probing the low-temperature chain-branching mechanism of $n$-butane autoignition chemistry via time-resolved measurements of ketohydroperoxide formation in photolytically initiated $\mathrm{n}-\mathrm{C} 4 \mathrm{H} 10$ oxidation. Proc. Combust. Inst. 2015, 35, 291-298.

(23) Savee, J.; Papajak, E.; Rotavera, B.; Huang, H.; Eskola, A.; Welz, O.; Sheps, L.; Taatjes, C.; Zádor, J.; Osborn, D. Direct observation and kinetics of a hydroperoxyalkyl radical (QOOH). Science 2015, 347, 643-646.

(24) Sharma, S.; Raman, S.; Green, W. H. Intramolecular hydrogen migration in alkylperoxy and hydroperoxyalkylperoxy radicals: Accurate treatment of hindered rotors. J. Phys. Chem. A 2010, 114, 5689-5701.

(25) Mohamed, S. Y.; Davis, A. C.; Al Rashidi, M. J.; Sarathy, S. M. High-pressure limit rate rules for $\alpha-\mathrm{H}$ isomerization of hydroperoxyalkylperoxy radicals. J. Phys. Chem. A 2018, 122, 3626-3639.

(26) Hoyermann, K.; Mauß, F.; Olzmann, M.; Welz, O.; Zeuch, T. Exploring the chemical kinetics of partially oxidized intermediates by combining experiments, theory, and kinetic modeling. Phys. Chem. Chem. Phys. 2017, 19, 18128-18146.

(27) Mohamed, S. Y.; Cai, L.; Khaled, F.; Banyon, C.; Wang, Z.; Al Rashidi, M. J.; Pitsch, H.; Curran, H. J.; Farooq, A.; Sarathy, S. M. Modeling ignition of a heptane isomer: Improved thermodynamics, reaction pathways, kinetics, and rate rule optimizations for 2-methylhexane. J. Phys. Chem. A 2016, 120, 22012217.

(28) Wang, Z.; Sarathy, S. M. Third $\mathrm{O} 2$ addition reactions promote the low-temperature autoignition of n-alkanes. Combust. Flame 2016, 165, 364-372.

(29) Wang, Z.; Zhang, L.; Moshammer, K.; Popolan-Vaida, D. M.; Shankar, V. S. B.; Lucassen, A.; Hemken, C.; Taatjes, C. A.; Leone, S. R.; Kohse-Höinghaus, K.; et al. Additional chain-branching pathways in the low-temperature oxidation of branched alkanes. Combust. Flame 2016, 164, 386-396.

(30) Silke, E. J.; Curran, H. J.; Simmie, J. M.; Pitz, W. J.; Westbrook, C. K. A Rapid Compression Machine Modelling Study of the Heptane Isomers. In European Combustion Meeting; Louvain-la-Neuve: Belgium, 2005.

(31) Wang, Z. D.; Popolan-Vaida, D. M.; Chen, B. J.; Moshammer, K.; Mohamed, S. Y.; Wang, H.; Sioud, S.; Raji, M. A.; Kohse-Hoinghaus, K.; Hansen, N.; et al. Unraveling the structure and chemical 
mechanisms of highly oxygenated intermediates in oxidation of organic compounds. Proc. Nat. Acad. Sci. U.S.A. 2017, 114, 13102-13107.

(32) Xing, L.; Bao, J. L.; Wang, Z.; Wang, X.; Truhlar, D. G. Hydrogen shift isomerizations in the kinetics of the second oxidation mechanism of alkane combustion. Reactions of the hydroperoxyalkylperoxy OOQOOH radical. Combust. Flame 2018, 197, 88-101.

(33) Goldsmith, C. F.; Green, W. H.; Klippenstein, S. J. Role of $\mathrm{O}_{2}+\mathrm{QOOH}$ in low-temperature ignition of propane. 1. Temperature and pressure dependent rate coefficients. J. Phys. Chem. A 2012, 116, 3325-3346.

(34) Zheng, J.; Truhlar, D. G. Quantum thermochemistry: Multistructural method with torsional anharmonicity based on a coupled torsional potential. J. Chem. Theory Comput. 2013, 9, 1356-1367.

(35) Zhao, Y.; Schultz, N. E.; Truhlar, D. G. Design of density functionals by combining the method of constraint satisfaction with parametrization for thermochemistry, thermochemical kinetics, and noncovalent interactions. J. Chem. Theory Comput. 2006, 2, 364-382.

(36) Papajak, E.; Truhlar, D. G. What are the most efficient basis set strategies for correlated wave function calculations of reaction energies and barrier heights? J. Chem. Phys. 2012, 137, 064110.

(37) Bao, J. L.; Zheng, J.; Truhlar, D. G. Kinetics of hydrogen radical reactions with toluene including chemical activation theory employing system-specific quantum RRK theory calibrated by variational transition state theory. J. Am. Chem. Soc. 2016, 138, 2690-2704.

(38) Bao, J. L.; Zhang, X.; Truhlar, D. G. Predicting pressure-dependent unimolecular rate constants using variational transition state theory with multidimensional tunneling combined with system-specific quantum RRK theory: a definitive test for fluoroform dissociation. Phys. Chem. Chem. Phys. 2016, 18, 16659-16670.

(39) Bao, J. L.; Truhlar, D. G. Silane-initiated nucleation in chemically active plasmas: validation of density functionals, mechanisms, and pressure-dependent variational transition state calculations. Phys. Chem. Chem. Phys. 2016, 18, 10097-10108.

(40) Bao, J. L.; Truhlar, D. G. Variational transition state theory: theoretical framework and recent developments. Chem. Soc. Rev. 2017, 46, 7548-7596.

(41) Zheng, J.; Mielke, S. L.; Clarkson, K. L.; Truhlar, D. G. MSTor: A program for calculating partition functions, free energies, enthalpies, entropies, and heat capacities of complex molecules including torsional anharmonicity. Comput. Phys. Commun. 2012, 183, 1803-1812.

(42) Zheng, J.; Meana-Pañeda, R.; Truhlar, D. G. MSTor - version 2013: A new version of the computer code for the multi-structural torsional anharmonicity, now with a coupled torsional potential. Comput. Phys. Commun. 2013, 184, 2032-2033.

(43) Bao, J. L.; Zheng, J.; Truhlar, D. G. Kinetics of hydrogen radical reactions with toluene including chemical activation theory employing system-specific quantum rrk theory calibrated by variational transition state theory. J. Am. Chem. Soc. 2016, 138, 2690-2704.

(44) Kassel, L. S. Kinetics of homogenous gas reactions; Chemical Catalog Co.: New York. 1932.

(45) Dean, A. M. Predictions of pressure and temperature effects upon radical addition and recombination reactions. J. Phys. Chem. 1985, 89, 4600-4608.

(46) Dean, A. M.; Westmoreland, P. R. Bimolecular QRRK analysis of methyl radical reactions. Int. J. Chem. Kinet. 1987, 19, 207-228.

(47) Chang, A. Y.; Bozzelli, J. W.; Dean, A. M. Kinetic analysis of complex chemical activation and unimolecular dissociation reactions using QRRK theory and the modified strong collision approximation. Z. Phys. Chem. 2000, 214, 1533-1568.

(48) Sheng, C. Y.; Bozzelli, J. W.; Dean, A. M.; Chang, A. Y. Detailed kinetics and thermochemistry of $\mathrm{C}_{2} \mathrm{H}_{5} \mathrm{O}_{2}$ : Reaction kinetics of the chemically-activated and stabilized $\mathrm{CH}_{3} \mathrm{CH}_{2} \mathrm{OO}$ - adduct. J. Phys. Chem. A 2002, 106, 7276-7293. 
(49) Frisch, M. J.; Trucks, G. W.; Schlegel, H. B.; Scuseria, G. E.; Robb, M. A.; Cheeseman, J. R.; Scalmani, G.; Barone, V.; Petersson, G. A.; Nakatsuji, H.; et al. Gaussian 16, Revision A.03, Gaussian, Inc., Wallingford CT, 2016.

(50) Zheng, J.; Mielke, S. L.; Bao, J. L.; Meana-Pañeda, R.; Clarkson, K. L.; Truhlar, D. G.; MSTor version 2017; University of Minnesota: Minneapolis, 2017. https://t1.chem.umn.edu/mstor/update.htm 1078 (accessed May 5, 2017).

(51) Liu, Y. P.; Lynch, G. C.; Truong, T. N.; Lu, D. H.; Truhlar, D. G.; Garrett, B. C. Molecular modeling of the kinetic isotope effect for the [1,5]-sigmatropic rearrangement of cis-1,3-pentadiene. J. Am. Chem. Soc. 1993, 115, 2408-2415.

(52) Garrett, B. C.; Truhlar, D. G. Criterion of minimum state density in the transition state theory of bimolecular reactions. J. Chem. Phys. 1979, 70, 1593-1598.

(53) Fernandez-Ramos, A.; Ellingson, B. A.; Garrett, B. C.; Truhlar, D. G. Variational transition state theory with multidimensional tunneling. Rev. Comput. Chem. 2007, 23, 125-232.

(54) Bao, J. L.; Meana-Paneda, R.; Truhlar, D. G. Multi-path variational transition state theory for chiral molecules: the site-dependent kinetics for abstraction of hydrogen from 2-butanol by hydroperoxyl radical, analysis of hydrogen bonding in the transition state, and dramatic temperature dependence of the activation energy. Chem. Sci. 2015, 6, 5866-5881.

(55) Alecu, I. M.; Zheng, J.; Zhao, Y.; Truhlar, D. G. Computational thermochemistry: Scale factor databases and scale factors for vibrational frequencies obtained from electronic model chemistries. $J$. Chem. Theory Comput. 2010, 6, 2872-2887.

(56) Bao, J. L.; Sripa, P.; Truhlar, D. G. Path-dependent variational effects and multidimensional tunneling in multi-path variational transition state theory: rate constants calculated for the reactions of $\mathrm{HO}_{2}$ with tert-butanol by including all 46 paths for abstraction at $\mathrm{C}$ and all six paths for abstraction at $\mathrm{O}$. Phys. Chem. Chem. Phys. 2016, 18, 1032-1041.

(57) Zheng, J.; Bao, J. L.; Meana-Pañeda, R.; Zhang, S.; Lynch, B. J.; Corchado, J. C.; Chuang, Y.-Y.; Fast, P. L.; Hu, W.-P.; Liu, Y.-P.; et al. Polyrate 2016-2A, University of Minnesota: Minneapolis, 2016, https://comp.chem.umn.edu/polyrate/.

(58) Zheng, J.; Bao, J. L.; Zhang, S.; Corchado, J. C.; Meana-Pañeda, R.; Chuang, Y.-Y.; Coitiño, E. L.; Ellingson, B. A.; Truhlar, D. G.; Gaussrate 2017, University of Minnesota: Minneapolis, 2017, https://comp.chem.umn.edu/gaussrate/.

(59) Page, M.; Mclver, J. W. On evaluating the reaction path Hamiltonian. J. Chem. Phys. 1988, 88, 922-935.

(60) Villa, J.; Truhlar, D. G. Variational transition state theory without the minimum-energy path. Theor. Chem. Acc. 1997, 97, 317-323.

(61) Jackels, C. F.; Gu, Z.; Truhlar, D. G. Reaction - path potential and vibrational frequencies in terms of curvilinear internal coordinates. J. Chem. Phys. 1995, 102, 3188-3201.

(62) Nguyen, K. A.; Jackels, C. F.; Truhlar, D. G. Reaction-path dynamics in curvilinear internal coordinates including torsions. J. Chem. Phys. 1996, 104, 6491-6496.

(63) Holbrook, K. A.; Pilling, M. J.; Robertson, S. H. Unimolecular Reactions; 2nd edn; John Wiley \& Sons: New York, 1996; p. 196.

(64) Lin, M. C.; Laidler, K. J. Theoretical analysis of gas-phase thermal isomerizations. Trans. Faraday Soc. 1968, 68, 94-102.

(65) Kiefer, J. H.; Kumaran, S. S.; Mudipalli, P. S. The mutual isomerization of allene and propene. Chem. Phys. Lett. 1994, 224, 51-55.

(66) Welty, J. R.; Wicks, C. E.; Wilson, R. E.; Rorrer, G. L. Fundamentals of Momentum Heat and Mass Transfer; John Wiley \& Sons: New York, 2001. 
(67) Xing, L.; Li, S.; Wang, Z.; Yang, B.; Klippenstein, S. J.; Zhang, F. Global uncertainty analysis for RRKM/master equation based kinetic predictions: A case study of ethanol decomposition. Combust. Flame 2015, 162, 3427-3436.

(68) Jasper, A. W.; Miller, J. A. Collisional energy transfer in unimolecular reactions: Direct classical trajectories for $\mathrm{CH}_{4} \rightleftarrows \mathrm{CH}_{3}+\mathrm{H}$ in helium. J. Phys. Chem. A 2009, 113, 5612-5619.

(69) Jasper, A. W.; Oana, C. M.; Miller, J. A. "Third-body" collision efficiencies for combustion modeling: Hydrocarbons in atomic and diatomic baths. Proc. Combust. Inst. 2015, 35, 197-204.

(70) Klippenstein, S. J. From theoretical reaction dynamics to chemical modeling of combustion. Proc. Combust. Inst. 2017, 36, 77-111.

(71) Gilbert, R. G.; Smith, S. C. Theory of Unimolecular and Recombination Reactions; Blackwell: Oxford, 1990.

(72) Adler, T. B.; Knizia, G.; Werner, H.-J. A simple and efficient CCSD(T)-F12 approximation. J. Chem. Phys. 2007, 127, 221106.

(73) Knizia, G.; Adler, T. B.; Werner, H.-J. Simplified CCSD(T)-F12 methods: Theory and benchmarks. J. Chem. Phys. 2009, 130, 054104.

(74) Zheng, J.; Yu, T.; Papajak, E.; Alecu, I. M.; Mielke, S. L.; Truhlar, D. G. Practical methods for including torsional anharmonicity in thermochemical calculations on complex molecules: The internalcoordinate multi-structural approximation. Phys. Chem. Chem. Phys. 2011, 13, 10885-10907.

(75) Wigner, E.

(76) Zheng, J.; Truhlar, D. G. Multi-path variational transition state theory for chemical reaction rates of complex polyatomic species: ethanol + $\mathrm{OH}$ reactions. Faraday Discuss. 2012, 157, 59-88.

(77) Barker, J. R.; Golden, D. M. Master equation analysis of pressure-dependent atmospheric reactions. Chem. Rev. 2003, 103, 4577-4592.

(78) Miller, J. A.; Pilling, M. J.; Troe, J. Unravelling combustion mechanisms through a quantitative understanding of elementary reactions. Proc. Combust. Inst. 2005, 30, 43-88.

(79) Fernández-Ramos, A.; Miller, J. A.; Klippenstein, S. J.; Truhlar, D. G. Modeling the kinetics of bimolecular reactions. Chem. Rev. 2006, 106, 4518-4584.

(80) Klippenstein, S. J.; Pande, V. S.; Truhlar, D. G. Chemical kinetics and mechanisms of complex systems: A perspective on recent theoretical advances. J. Am. Chem. Soc. 2014, 136, 528-546.

(81) Barker, J. R.; Ortiz, N. F. Multiple-well, multiple-path unimolecular reaction systems. II. 2methylhexyl free radicals. Int. J. Chem. Kinet. 2001, 33, 246-261.

(82) Wang, Z.; Zhang, L.; Moshammer, K.; Popolan-Vaida, D. M.; Shankar, V. S. B.; Lucassen, A.; Hemken, C.; Taatjes, C. A.; Leone, S. R.; Kohse-Höinghaus, K.; et al. Additional chain-branching pathways in the low-temperature oxidation of branched alkanes. Combust. Flame 2016, 164, 386-396. 
TOC graphic

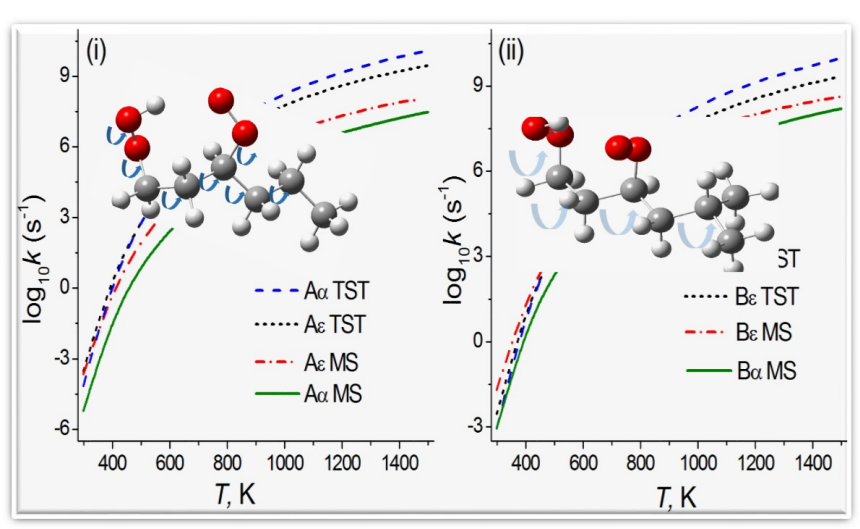

